TRANSACTIONS OF THE

AMERICAN MATHEMATICAL SOCIETY

Volume 361, Number 7, July 2009, Pages 3501-3533

S 0002-9947(09)04521-8

Article electronically published on March 4, 2009

\title{
SUPERCHARACTER FORMULAS FOR PATTERN GROUPS
}

\author{
PERSI DIACONIS AND NATHANIEL THIEM
}

\begin{abstract}
C. Andre and N. Yan introduced the idea of a supercharacter theory to give a tractable substitute for character theory in wild groups such as the unipotent uppertriangular group $U_{n}\left(\mathbb{F}_{q}\right)$. In this theory superclasses are certain unions of conjugacy classes, and supercharacters are a set of characters which are constant on superclasses. This paper gives a character formula for a supercharacter evaluated at a superclass for pattern groups and more generally for algebra groups.
\end{abstract}

\section{INTRODUCTION}

Let $U_{n}\left(\mathbb{F}_{q}\right)$ be the group of uppertriangular $n \times n$ matrices with entries in the finite field $\mathbb{F}_{q}$, and with ones on the diagonal. While describing the conjugacy classes or irreducible characters of $U_{n}\left(\mathbb{F}_{q}\right)$ is a well-known wild problem, Carlos André [1, 2, 4] and Ning Yan [17, 18, have shown that certain unions of conjugacy classes (hereinafter superclasses) and certain characters (hereinafter supercharacters) have an elegant theory that is rich enough to handle some Fourier analysis problems classically needing the full character table and yet tractable enough to admit a closed form formula for a supercharacter at a superclass (see also [8]).

Diaconis and Isaacs 10 abstracted supercharacter theory to algebra groups, a class of groups of the form $\{1+X \mid X \in \mathfrak{n}\}$, where $\mathfrak{n}$ is a nilpotent $\mathbb{F}_{q}$-algebra. In the resulting theory, the restriction of supercharacters and tensor products of supercharacters decompose as nonnegative integer combinations of supercharacters. Furthermore, there is a notion of superinduction that is dual to restriction of supercharacters. However, instead of giving a nice formula as in the case of $U_{n}\left(\mathbb{F}_{q}\right)$, they leave the character formula in the form of an orbit sum.

The present paper combines the above two perspectives by viewing algebra groups as subgroups of $U_{n}\left(\mathbb{F}_{q}\right)$. The primary focus of this paper is on a large class of subgroups called pattern subgroups. Let $J \subseteq\{(i, j) \mid 1 \leq i<j \leq n\}$ be a subset that is closed in the sense that $(i, j),(j, k) \in J$ implies $(i, k) \in J$ (i.e., $J$ is a partial order on $\{1,2, \ldots, n\})$. The pattern group $U_{J}$ is the subgroup of $U_{n}\left(\mathbb{F}_{q}\right)$ consisting of matrices whose $(i, j)$ th entry is permitted to be nonzero only if $(i, j) \in J$. In fact, pattern groups are type A root subgroups, and C. André and A. Neto have constructed a supercharacter theory for types B, C, and D [5].

We give a reasonably explicit formula for a supercharacter evaluated at a superclass for an arbitrary algebra group. It is, however, easiest to state for the special

Received by the editors October 5, 2006 and, in revised form, March 1, 2007.

2000 Mathematics Subject Classification. Primary 20C99, 05Exx.

Key words and phrases. Supercharacters, superclasses, finite unipotent group, algebra group, posets.

(C)2009 American Mathematical Society Reverts to public domain 28 years from publication 
case of pattern groups. Both supercharacters and superclasses can be indexed by certain (different) subsets of $U_{J}$. For $x, y, \in U_{J}$, let $\chi^{y}$ be the supercharacter corresponding to $y$; then there exists an explicit $|J| \times|J|$ matrix $M$ and vectors $a, b \in \mathbb{F}_{q}^{|J|}$ depending on $x$ and $y$ such that

$$
\begin{aligned}
& \overline{\chi^{y}(x)} \\
& = \begin{cases}\frac{\chi^{y}(1)}{q^{\operatorname{rank}(M)}} \prod_{(i, j)} \theta\left(b_{i j}^{\prime} b_{i j}+x_{i j} y_{i j}\right) & \text { if } M b^{\prime}=a \text { and the product is well defined, } \\
0, & \text { otherwise, }\end{cases}
\end{aligned}
$$

where $\theta: \mathbb{F}_{q} \rightarrow \mathbb{C}^{\times}$is an isomorphism. See Theorem 5.1 and Theorem 6.1 for explicit statements. This formula reduces to the André/Yan formula in the case when $J=\{(i, j) \mid 1 \leq i<j \leq n\}$, and to the usual character formula for the Heisenberg group in the case when $J=\{(i, j) \mid i=1$ or $j=n\}$.

Section 2 gives some background on pattern groups, algebra groups and supercharacter theory. We also characterize which subgroups of $U_{n}\left(\mathbb{F}_{q}\right)$ are algebra groups. Section 3 sets out our notation for pattern groups using the language of closed sets of roots. Section 4 describes the orbits and coorbits of an algebra group on its radical $\mathfrak{n}$, and dual $\mathfrak{n}^{*}$. This amounts to a careful study of row and column operations.

The main results are in Section 5, giving a formula in the form (1.1) for pattern groups. If the partial order given by $J$ has no chains of length four, then we show that superclasses and supercharacters are conjugacy classes and irreducible characters. In particular, our formulas give the classical characters of the extra special $p$-groups and certain unipotent radicals of Levi subgroups. At the other extreme, our formulas reduce to the André-Carter formulae for $U_{n}\left(\mathbb{F}_{q}\right)$. Several other examples are also given explicitly.

Section 6 shows that the character formula also holds for general algebra subgroups of pattern groups (and thus for all algebra groups).

\section{BACKGROUND}

This section gives an overview and pointers to the literature on three fundamental topics: pattern groups, algebra groups and supercharacters.

\subsection{Pattern groups. Let}

$U_{n}\left(\mathbb{F}_{q}\right)=\left\{n \times n\right.$ unipotent upper-triangular matrices with entries in $\left.\mathbb{F}_{q}\right\}$.

A closed subset of pairs

$$
J \subseteq\{(i, j) \mid 1 \leq i<j \leq n\}
$$

is a subset with the property that $(i, j),(j, k) \in J$ implies $(i, k) \in J$. For $t \in \mathbb{F}_{q}$, let $x_{i j}(t) \in U_{n}\left(\mathbb{F}_{q}\right)$ denote the matrix with ones on the diagonal, $t$ in the $(i, j)$ th position and zeroes everywhere else. Then for any closed subset $J \subseteq\{(i, j) \mid 1 \leq$ $i<j \leq n\}$, the pattern group $U_{J}$ is

$$
\begin{aligned}
U_{J} & =\left\{\left(u_{i j}\right) \in U_{n}\left(\mathbb{F}_{q}\right) \mid(i, j) \notin J \text { implies } u_{i j}=0\right\} \\
& =\left\langle x_{i j}(t) \mid t \in \mathbb{F}_{q},(i, j) \in J\right\rangle .
\end{aligned}
$$


The closedness of $J$ implies that $U_{J}$ is a subgroup of $U_{n}\left(\mathbb{F}_{q}\right)$. It is also clear that

$$
\left|U_{J}\right|=q^{|J|} \text {. }
$$

Note that the set $J$ gives rise to a poset $\mathcal{P}$ on $\{1,2, \ldots, n\}$, where $i \leq_{\mathcal{P}} j$ if and only if $(i, j) \in J$. The transitivity property of $\mathcal{P}$ is the same as the closed property of $J$. In the language of posets, these pattern groups are similar to Rota's incidence algebras [16.

Alternatively, if $R^{+}=\left\{\varepsilon_{i}-\varepsilon_{j} \mid 1 \leq i<j \leq n\right\}$ are the usual positive roots of type $A$, then $J \subseteq R^{+}$is closed if and only if $\alpha, \beta \in J$ and $\alpha+\beta \in R^{+}$implies $\alpha+\beta \in J$. From this point of view $U_{J}$ is the subgroup of $U_{n}\left(\mathbb{F}_{q}\right)$ generated by the one-parameter subgroups $\left\langle x_{\alpha}(t) \mid t \in \mathbb{F}_{q}\right\rangle$ corresponding to $\alpha \in J$.

Examples. Many naturally occurring subgroups are pattern groups.

(1) If $J=\{(i, j) \mid 1 \leq i<j \leq n\}$, then the corresponding poset is

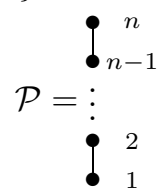

and $U_{J}=U_{n}\left(\mathbb{F}_{q}\right)$.

(2) The commutator subgroup $U_{n}^{\prime} \subseteq U_{n}\left(\mathbb{F}_{q}\right)$ is equal to the Frattini subgroup $\Phi\left(U_{n}\right) \subseteq U_{n}\left(\mathbb{F}_{q}\right)$. In this case, $U_{n}^{\prime}=\Phi\left(U_{n}\right)=U_{J}$, where

$$
J=\{(i, j) \mid 1 \leq i<i+1<j \leq n\}, \quad \text { or }
$$

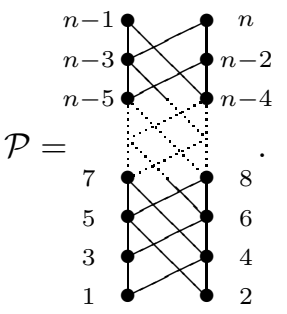

In general, if $J$ is closed, then $U_{J}^{\prime}=\Phi\left(U_{J}\right)=U_{J^{\prime}}$ 11, where

$$
J^{\prime}=\{(i, k) \mid(i, j),(j, k) \in J, \text { for some } j\} .
$$

Let $\left\{t_{1}, t_{2}, \ldots, t_{r}\right\}$ be a basis for $\mathbb{F}_{q}$ as an $\mathbb{F}_{p}$-vector space (where $q=$ $p^{r}$ ). From the description of the Frattini subgroups, a minimal (Frattini) generating set for $U_{J}$ may be chosen as

$$
\left\{x_{i j}(t) \mid(i, j) \in J, t \in\left\{t_{1}, t_{2}, \ldots, t_{r}\right\}, i \leq k \leq j \text { implies }(i, k) \notin J \text { or }(k, j) \notin J\right\} \text {. }
$$

Note that the set of $(i, j)$ that come up in these generators is the set of covering relations of the corresponding poset $\mathcal{P}$.

(3) The center of $U_{n}\left(\mathbb{F}_{q}\right)$ is $U_{J}$ for $J=\{(1, n)\}$, or

$$
\mathcal{P}={ }_{1}^{n} \bullet \quad \bullet \quad \bullet \quad \cdots \bullet
$$

(4) The upper and lower central series of $U_{n}\left(\mathbb{F}_{q}\right)$ have terms given by the pattern groups

$$
J_{k}=\{(i, j) \mid j-i \geq k\} .
$$

(5) In $U_{n}\left(\mathbb{F}_{q}\right)$, Vera-Lopez and Arregi [7] used a sequence of subgroups given by

$$
G_{i j}=\left\langle x_{k l}(t) \mid t \in \mathbb{F}_{q}, i \leq k, j \leq l,(i, j) \neq(k, l)\right\rangle .
$$


In this case,

$$
1=G_{1 n} \triangleleft G_{1, n-1} \triangleleft \cdots \triangleleft G_{12} \triangleleft G_{2 n} \triangleleft \cdots \triangleleft G_{n-1, n} \triangleleft U_{n}\left(\mathbb{F}_{q}\right)
$$

gives a central series for $U_{n}\left(\mathbb{F}_{q}\right)$ with each factor isomorphic to $\mathbb{F}_{q}^{+}$. Furthermore, every subgroup is a pattern group.

The following elegant characterization of pattern groups was communicated to us by Bob Guralnick.

Proposition 2.1. For $q \geq 3$, a subgroup $U$ of $U_{n}\left(\mathbb{F}_{q}\right)$ is a pattern group if and only if $U$ is invariant under conjugation by diagonal matrices $T$ in the general linear group $G L_{n}\left(\mathbb{F}_{q}\right)$.

Proof. Note that

$$
\operatorname{diag}\left(t_{1}, t_{2}, \ldots, t_{n}\right)\left(u_{i j}\right) \operatorname{diag}\left(t_{1}^{-1}, t_{2}^{-1}, \ldots, t_{n}^{-1}\right)=\left(t_{i} u_{i j} t_{j}^{-1}\right) .
$$

Thus, if $U$ is a pattern group, then it is invariant under the action of $T$.

Suppose $U$ is invariant under $T$. Let $u=\left(u_{i j}\right) \in U$ be nontrivial. It suffices to show that for every $u_{i j} \neq 0$, the group $\left\langle x_{i j}(t) \mid t \in \mathbb{F}_{q}\right\rangle$ is in $U$. Let $i$ be minimal so that $u_{i j} \neq 0$ for some $i<j \leq n$. Let

$$
h_{i}(t)=\operatorname{diag}(\underbrace{1, \ldots, 1}_{i-1}, t, 1, \ldots, 1) .
$$

Then $h_{i}(t) u h_{i}\left(t^{-1}\right) \in U$ has the effect of multiplying the $i$ th row of $u$ by $t$. In particular, if $t \neq 1$, then

$$
u^{\prime}=h_{i}(t) u h_{i}\left(t^{-1}\right) u^{-1} \in U \quad \text { satisfies } \quad \text { for } j<k, u_{j k}^{\prime}=0 \quad \text { unless } j=i .
$$

Now let $j$ be minimal such that $u_{i j}^{\prime} \neq 0$ (such a $j$ must exist by our choice of $i$ ). Then $h_{j}\left(t_{2}\right) u^{\prime} h_{j}\left(t_{2}^{-1}\right) \in U$ has the effect of multiplying the $(i, j)$ th entry by $t_{2}^{-1}$. Thus, if $t_{2} \neq 1$,

$$
u^{\prime \prime}=h_{j}\left(t_{2}\right) u^{\prime} h_{j}\left(t_{2}^{-1}\right) u^{\prime-1} \in U \quad \text { satisfies } \quad u_{k l}^{\prime \prime}=0 \quad \text { unless } k=i \text { and } l=j .
$$

Thus, $u^{\prime \prime}=x_{i j}(t)$ for some $t \in \mathbb{F}_{q}^{\times}$. Since $U$ is invariant under $T$,

$$
\left\langle h_{i}\left(t_{3}\right) x_{i j}(t) h_{i}\left(t_{3}^{-1}\right) \mid t_{3} \in \mathbb{F}_{q}^{\times}\right\rangle=\left\langle x_{i j}\left(t_{3} t\right) \mid t_{3} \in \mathbb{F}_{q}^{\times}\right\rangle=\left\langle x_{i j}(t) \mid t \in \mathbb{F}_{q}\right\rangle \subseteq U .
$$

Note that $u^{\prime \prime \prime}=u x_{i j}\left(-u_{i j}\right)$ satisfies $u_{i j}^{\prime \prime \prime}=0$ and $u_{k l}^{\prime \prime \prime}=u_{k l}$ for all $(k, l) \neq(i, j)$. We may therefore proceed inductively to find all the one-parameter subgroups in $U$.

Remarks. (1) The proof fails for $q=2$ because the proof requires $\left|\mathbb{F}_{q}^{\times}\right|>1$. In fact, if $q=2$ the diagonal condition is empty, and the proposition is false. For example,

$$
\left\{\left(\begin{array}{ccc}
1 & s & t \\
0 & 1 & s \\
0 & 0 & 1
\end{array}\right) \mid s, t \in \mathbb{F}_{2}\right\} \subseteq U_{3}\left(\mathbb{F}_{2}\right)
$$

is a subgroup but not a pattern group since it does not contain $x_{12}(1)$.

(2) Proposition 2.1 implies all characteristic subgroups of $U_{n}\left(\mathbb{F}_{q}\right)$ are pattern subgroups for $q \geq 3$. The subgroup in Remark 1 is characteristic, so the $q=2$ case also fails here.

(3) The proof does not depend on the field (other than the size constraint). 
2.2. Algebra groups. Algebra groups generalize pattern groups. Let $\mathfrak{n}$ be a finite dimensional nilpotent $\mathbb{F}_{q}$-algebra. The corresponding algebra group $U_{\mathfrak{n}}$ is the group

$U_{\mathfrak{n}}=\{1+X \mid X \in \mathfrak{n}\}$ with multiplication given by $(1+X)(1+Y)=1+X+Y+X Y$.

Algebra groups have $q$-power order, and in [15] Robinson studied the number of conjugacy classes of an algebra group. It is obvious that the center of an algebra group is an algebra group since $Z\left(U_{\mathfrak{n}}\right)=1+Z(\mathfrak{n})$. We do not know if the Frattini subgroups of an algebra group are algebra groups, but the commutator subgroup of an algebra group is not always an algebra group [12. The classification of nilpotent algebras is an impossible task, but many classes of examples are known (see Pierce [14). In this section we show that algebra groups are poset groups.

By Engels theorem we may view $\mathfrak{n}$ as a subalgebra of the set of $n \times n$ uppertriangular matrices with zeroes on the diagonal (for some suitably chosen $n$ ), so every algebra group is isomorphic to a subgroup of $U_{n}\left(\mathbb{F}_{q}\right)$. The following proposition characterizes which subgroups of $U_{n}\left(\mathbb{F}_{q}\right)$ are algebra groups. Indeed, we determine which subgroups of $U_{J}$ are algebra groups for general closed sets $J$.

Let $J^{*}=\left\{\phi: J \rightarrow \mathbb{F}_{q}\right\}$. For $\phi \in J^{*}$, let

$$
x_{\phi}=\left(\begin{array}{ccc}
1 & & \phi(i, j) \\
& \ddots & \\
0 & & 1
\end{array}\right) \in U_{J} \text { and } X_{\phi}=\left(\begin{array}{ccc}
0 & & \phi(i, j) \\
& \ddots & \\
0 & & 0
\end{array}\right) \in \mathfrak{n}_{J}=U_{J}-1 .
$$

Proposition 2.2. For closed $J$, let $H \subseteq U_{J}$ be a subgroup. Then

$$
V=\left\{\phi \in J^{*} \mid x_{\phi} \in H\right\}
$$

is an $\mathbb{F}_{q}$-vector space if and only if $H$ is a subalgebra group of $U_{J}$.

Proof. Suppose $V$ is a vector space. It suffices to show

$$
\mathfrak{n}_{H}=\left\{X_{\phi} \mid \phi \in V\right\}
$$

is an $\mathbb{F}_{q^{-}}$-algebra. Note that

$$
a X_{\phi}+b X_{\rho} \in \mathfrak{n}_{H}
$$

is equivalent to $V$ being a vector space. Consider

$$
X_{\phi} X_{\rho}=\left(x_{\phi}-1\right)\left(x_{\rho}-1\right)=x_{\phi} x_{\rho}-x_{\phi}-x_{\rho}+1 .
$$

Since $x_{\phi} x_{\rho} \in H_{J}, x_{\phi} x_{\rho}=x_{\eta}$ for some $\eta \in V$. Thus,

$$
X_{\phi} X_{\rho}=X_{\eta-\phi-\rho} \in \mathfrak{n}_{H} .
$$

Suppose $H_{J}$ is a subalgebra group of $U_{J}$. If $t_{\rho}, t_{\mu} \in V$ and $a, b \in \mathbb{F}_{q}$, then

$$
1+\left(a X_{\rho}+b X_{\mu}\right) \in H_{J}
$$

implies that $a \rho+b \mu \in V$.

Remarks. (1) Suppose $H \subseteq U_{n}\left(\mathbb{F}_{q}\right)$ is an algebra group. Then there is a natural poset $\mathcal{P}$ on $\{1,2, \ldots, n\}$ given by $i \leq_{\mathcal{P}} k$ if either

(a) there exists $x_{\phi} \in H$ such that $\phi_{i k} \neq 0$,

(b) there exist $x_{\phi}, x_{\rho} \in H$ such that $\phi_{i j} \neq 0$ and $\rho_{j k} \neq 0$ for some $j \in$ $\{i+1, i+2, \ldots, k-1\}$. 
If $J=\left\{(i, j) \in \mathcal{P} \times \mathcal{P} \mid i<_{\mathcal{P}} j\right\}$, then $U_{J}$ is the smallest pattern subgroup of $U_{n}\left(\mathbb{F}_{q}\right)$ that contains $H$. For example, if $H$ is the algebra subgroup

$$
H=\left\{\left(\begin{array}{cccc}
1 & a & a & 0 \\
0 & 1 & 0 & -a \\
0 & 0 & 1 & a \\
0 & 0 & 0 & 1
\end{array}\right) \mid a \in \mathbb{F}_{q}\right\} \subseteq U_{4}\left(\mathbb{F}_{q}\right),
$$

then the corresponding poset is

$$
\mathcal{P}=2 \nearrow_{1}^{4} \backslash 3 \text { with pattern group } U_{J}=\left\{\left(\begin{array}{cccc}
1 & a & c & e \\
0 & 1 & 0 & d \\
0 & 0 & 1 & b \\
0 & 0 & 0 & 1
\end{array}\right) \mid a, b, c, d, e \in \mathbb{F}_{q}\right\} .
$$

(2) Not every $p$-group is an algebra group. In fact, one can use Proposition 2.2 to show that the maximal unipotent subgroup of $\operatorname{Sp}_{2 n}\left(\mathbb{F}_{q}\right)$ is not an algebra group (for $n>1$ ). In this way, algebra groups are a "type A" phenomenon. However, every $p$-group is a subgroup of some algebra group.

(3) Much of the theory can be adapted when we replace $\mathbb{F}_{q}$ by a finite radical ring, as explored in [6].

2.3. Supercharacters for algebra groups. Determining conjugacy classes and characters of $p$-groups contains intractable obstacles. For $U_{n}\left(\mathbb{F}_{q}\right)$, André 1 , 2, 4, Yan [17] and Arias-Castro et al [8] found that certain unions of conjugacy classes and corresponding sums of irreducible characters give a tractable, useful theory. This was abstracted to algebra groups in [10. We give a brief synopsis.

First suppose that $G$ is an arbitrary finite group. A supercharacter theory for $G$ is a partition $\kappa$ of the conjugacy classes, and a partition $\kappa^{\vee}$ of the irreducible characters such that

(a) the identity element is in its own block in $\kappa$,

(b) $|\kappa|=\left|\kappa^{\vee}\right|$,

(c) for each block $K \in \kappa^{\vee}$, there exists a corresponding character $\chi^{K}$ which is a positive linear combination of the characters in $K$ such that $\chi^{K}$ is constant on the blocks of $\kappa$.

There are many general examples of supercharacter theories, including the partitioning of $G$ and its characters under the action of a group of automorphisms of $G$. The following specific construction for algebra groups specializes to the construction of André-Yan (see [10]).

If $U_{\mathfrak{n}}$ is an algebra group with corresponding nilpotent algebra $\mathfrak{n}$, then $U_{\mathfrak{n}} \times U_{\mathfrak{n}}$ acts on $\mathfrak{n}$ by left and right multiplication. A superclass of $U_{\mathfrak{n}}$ is a subset $1+O$, where $O$ is a two-sided orbit in $\mathfrak{n}$. Note that every superclass is in fact a union of conjugacy classes and 1 is in its own superclass.

To define supercharacters, note that $U_{\mathfrak{n}} \times U_{\mathfrak{n}}$ acts on the space of linear functionals $\mathfrak{n}^{*}$ by

$$
(u \lambda v)(X)=\lambda\left(u^{-1} X v^{-1}\right), \quad \text { where } u, v \in U_{\mathfrak{n}}, \lambda \in \mathfrak{n}^{*}, \text { and } X \in \mathfrak{n} .
$$

Fix a nontrivial homomorphism $\theta: \mathbb{F}_{q}^{+} \rightarrow \mathbb{C}^{\times}$. For $\lambda \in \mathfrak{n}^{*}$, define the supercharacter $\chi^{\lambda}$ to be

$$
\chi^{\lambda}=\frac{\left|\lambda U_{\mathfrak{n}}\right|}{\left|U_{\mathfrak{n}} \lambda U_{\mathfrak{n}}\right|} \sum_{\mu \in U_{\mathfrak{n}} \lambda U_{\mathfrak{n}}} \theta \circ \mu
$$


Then every irreducible character appears as a constituent of exactly one supercharacter (not obvious), and the supercharacters are constant on superclasses (follows from the definition).

The classical orbit method of Kirillov attempts to identify conjugacy classes and irreducible characters with orbits of $U_{\mathfrak{n}}$ acting on $\mathfrak{n}$ by conjugation, but requires that the characteristic of $\mathbb{F}_{q}$ be large. Another problem with this construction is that describing the orbits is a provably wild problem. André and Yan have shown that the $U_{\mathfrak{n}} \times U_{\mathfrak{n}}$-orbits for $U_{n}\left(\mathbb{F}_{q}\right)$ are indexed by labeled set partitions and that natural quantities such as dimensions and intertwining numbers are described in terms of elegant combinatorics (rivaling the tableaux combinatorics of the symmetric groups). We hope that the following developments show that some of this carries over to general algebra groups.

Diaconis and Isaacs describe in [10, that the above superclasses and supercharacters for algebra groups form a "nice" supercharacter theory in the following sense.

(a) The character of the regular representation is the sum of all the distinct supercharacters. In particular, supercharacters are orthogonal with respect to the usual inner product.

(b) The restriction of a supercharacter to any algebra subgroup is a sum of supercharacters with nonnegative integer coefficients.

(c) There is a notion of "superinduction" for which Frobenius reciprocity holds.

(d) The tensor product of supercharacters is a linear combination of supercharacters with nonnegative integer coefficients.

In addition, [10] give useful criteria for determining when a superclass or supercharacter is in fact a conjugacy class or irreducible character. We apply this in Corollary 5.2 to give a simple necessary and sufficient condition for a supercharacter of $U_{n}\left(\mathbb{F}_{q}\right)$ to be irreducible.

The theory is rich enough to permit analysis of natural problems. For example, consider a pattern group $U_{J}$. Fix a basis $\left\{t_{1}, \ldots, t_{r}\right\}$ of $\mathbb{F}_{q}$ as an $\mathbb{F}_{p}$-vector space $\left(q=p^{r}\right)$. Then the set

$$
\left\{x_{i j}(t) \mid t \in\left\{t_{1}, \ldots, t_{r}\right\},(i, j) \in J, \text { there is no pair }(i, k),(k, j) \in J\right\}
$$

is a generating set for $U_{J}$. It follows from [10, Corollary 3.5] that the conjugacy class containing one of these generators is a superclass. Thus, supercharacter theory can be used to explicitly diagonalize the conjugacy walk on $U_{J}$. Then one may attempt comparison theory [11] to analyze the original walk. This program was carried out in [8] for $U_{n}\left(\mathbb{F}_{q}\right)$.

\section{PATtern GRoups}

This section gives two useful orderings on pairs $(i, j)$ and defines pattern groups.

\subsection{A Lie-theoretic perspective. Let}

$$
R^{+}=\left\{\varepsilon_{i}-\varepsilon_{j} \mid 1 \leq i<j \leq n\right\}
$$

be a set of roots, which has a total order given by

$$
\varepsilon_{r}-\varepsilon_{s} \leq \varepsilon_{i}-\varepsilon_{j}, \quad \text { if } r>i \text {, or if } r=i \text { and } s>j .
$$

If the roots are indexed by pairs $(i, j)$, then this ordering can be pictured as

$$
(n-1, n)<(n-2, n)<(n-2, n-1)<\cdots<(1, n)<(1, n-1)<\cdots<(1,2) .
$$


Lemma 3.1. The total order $\leq$ satisfies

(a) If $\alpha<\beta$ and $\alpha+\beta \in R^{+}$, then $\alpha<\alpha+\beta<\beta$.

(b) If $\alpha<\beta, \alpha+\beta \in R^{+}$and $\alpha+\beta+\gamma \in R^{+}$, then either $\gamma<\alpha$ or $\beta<\gamma$.

Proof. (a) The assumptions imply $\alpha=\varepsilon_{j}-\varepsilon_{k}, \beta=\varepsilon_{i}-\varepsilon_{j}$, so $\alpha+\beta=\varepsilon_{i}-\varepsilon_{k}$. Since $j>i, \alpha<\alpha+\beta$ and since $k>j, \alpha+\beta<\beta$. (b) The assumptions imply that $\alpha=\varepsilon_{j}-\varepsilon_{k}, \beta=\varepsilon_{i}-\varepsilon_{j}$, and either $\gamma=\varepsilon_{h}-\varepsilon_{i}$ or $\gamma=\varepsilon_{k}-\varepsilon_{l}$.

There is also a partial ordering on the roots given by

$$
\alpha \prec \beta \quad \text { if } \beta-\alpha \in R^{+},
$$

called the dominance order. This ordering can be pictured by hanging the upper triangle from its right-hand corner $(1, n)$. Entries just above the main diagonal are lowest, and those on the second diagonal are above with $(1, n)$ at the top of the Hasse diagram (see the figure below). This partial ordering is not compatible with the linear ordering 3.1, and should also not be confused with the underlying poset $\mathcal{P}$ on the integers $\{1,2, \ldots, n\}$.

Let $\mathcal{G}\left(R^{+}\right)$be the Haase diagram of the poset $\preceq$, which we can organize from smallest to greatest as we pass from left to right. For example, if $n=4$,

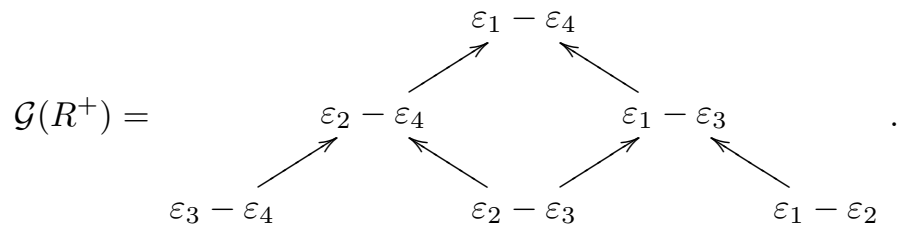

A subset $J \subseteq R^{+}$is closed if $\alpha, \beta \in J$ implies that $\alpha+\beta \in J$. Let $\mathcal{G}(J)$ denote the subgraph of $\mathcal{G}\left(R^{+}\right)$with vertices $J$ and an edge from $\alpha$ to $\beta$ if $\beta-\alpha \in J$. For example, when $n=4$, the subset $J=\{(1,2),(1,3),(1,4),(2,4),(3,4)\}$ is closed with corresponding subgraph

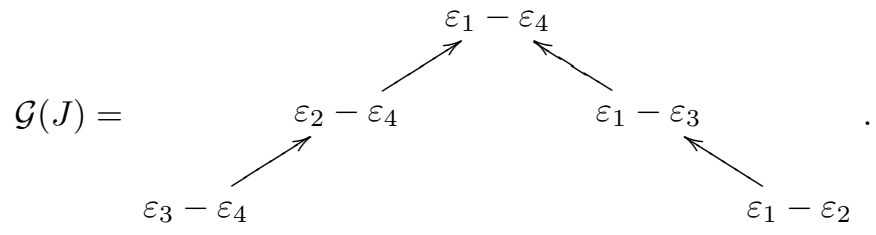

3.2. Pattern groups. For $\varepsilon_{i}-\varepsilon_{j} \in R^{+}$, let

$X_{i j}=X_{\varepsilon_{i}-\varepsilon_{j}}=n \times n$ matrix with 1 in the $(i, j)$ th position and zeroes elsewhere.

The nilpotent $\mathbb{F}_{q^{-}}$algebra

$$
\mathfrak{n}_{J}=\mathbb{F}_{q^{-}} \operatorname{span}\left\{X_{\alpha} \mid \alpha \in J\right\}
$$

has relations

$$
\begin{aligned}
X_{\alpha}^{2} & =0, & & \text { for } \alpha \in J, \\
X_{\alpha} X_{\beta} & =0, & & \text { for } \alpha, \beta \in J \text { such that } \alpha<\beta \text { OR } \alpha+\beta \notin R^{+}, \\
X_{\beta} X_{\alpha} & =X_{\alpha+\beta} & & \text { for } \alpha, \beta, \alpha+\beta \in J, \alpha<\beta \text { AND } \alpha+\beta \in R^{+} .
\end{aligned}
$$


Let $J \subseteq R^{+}$be closed, let $\mathbb{F}_{q}$ be the finite field with $q$ elements, and let

$$
J^{*}=\left\{\begin{array}{rlc}
\phi: J & \rightarrow & \mathbb{F}_{q} \\
\alpha & \mapsto & \phi_{\alpha}
\end{array}\right\} .
$$

Then

$$
\mathfrak{n}_{J}=\left\{X_{\phi} \mid \phi \in J^{*}\right\} \quad \text { where } \quad X_{\phi}=\sum_{\alpha \in J} \phi_{\alpha} X_{\alpha} .
$$

The dual of $\mathfrak{n}_{J}$,

$$
\mathfrak{n}_{J}^{*}=\left\{\lambda: \mathfrak{n}_{J} \rightarrow \mathbb{F}_{q} \mid \lambda \mathbb{F}_{q} \text {-linear }\right\},
$$

has a basis $\left\{\lambda_{\alpha}: \mathfrak{n}_{J} \rightarrow \mathbb{F}_{q} \mid \alpha \in J\right\}$ given by

$$
\lambda_{\alpha}\left(X_{\phi}\right)=\phi_{\alpha} .
$$

For $\eta \in J^{*}$, let

$$
\lambda_{\eta}=\sum_{\alpha \in J} \eta_{\alpha} \lambda_{\alpha}
$$

so that $\mathfrak{n}_{J}^{*}=\left\{\lambda_{\eta} \mid \eta \in J^{*}\right\}$.

For $\alpha \in J$ and $t \in \mathbb{F}_{q}$, define

$$
x_{\alpha}(t)=e^{t X_{\alpha}}=1+t X_{\alpha} .
$$

Note that if we order the product by the total order $<$ (i.e., begin with the smallest, multiply on the right by the next smallest, etc.), then relation (3.3) implies

$$
\prod_{\alpha \in J} x_{\alpha}\left(t_{\alpha}\right)=1+\sum_{\alpha \in J} t_{\alpha} X_{\alpha}
$$

so we may define the unipotent subgroup

$$
\begin{aligned}
U_{J} & =\left\langle x_{\alpha}(t) \quad \mid \alpha \in J, t \in \mathbb{F}_{q}\right\rangle \\
& =1+\mathfrak{n}_{J} .
\end{aligned}
$$

For $\phi \in J^{*}$, let

$$
x_{\phi}=1+X_{\phi}=\prod_{\alpha \in J} x_{\alpha}\left(\phi_{\alpha}\right) \in U_{J},
$$

where again the product is ordered according to $<$. Then

$$
U_{J}=\left\{x_{\phi} \mid \phi \in J^{*}\right\} \quad \text { and } \quad \mathfrak{n}_{J}=\left\{X_{\phi} \mid \phi \in J^{*}\right\} .
$$

The generators of $U_{J}$ satisfy the commutation relation

$$
\left[x_{\alpha}(a), x_{\beta}(b)\right]= \begin{cases}x_{\alpha+\beta}(-a b), & \text { if } \alpha+\beta \in J, \alpha<\beta, \\ x_{\alpha+\beta}(a b), & \text { if } \alpha+\beta \in J, \alpha>\beta, \\ 1, & \text { otherwise }\end{cases}
$$

and the additive relation

$$
x_{\alpha}(a) x_{\alpha}(b)=x_{\alpha}(a+b) .
$$

Example. To connect previous poset notation with the root graph, consider the poset of nonempty subsets on three elements,

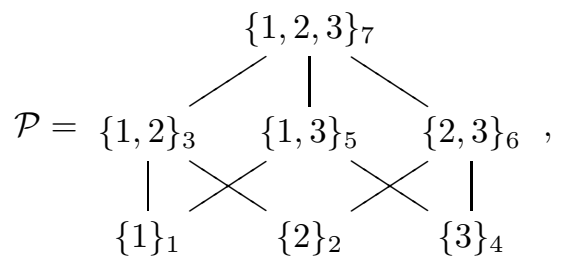


so that $J=\{(6,7),(5,7),(4,7),(4,6),(4,5),(3,7),(2,7),(2,6),(2,3),(1,7),(1,5)$, $(1,3)\}$. The corresponding root graph is

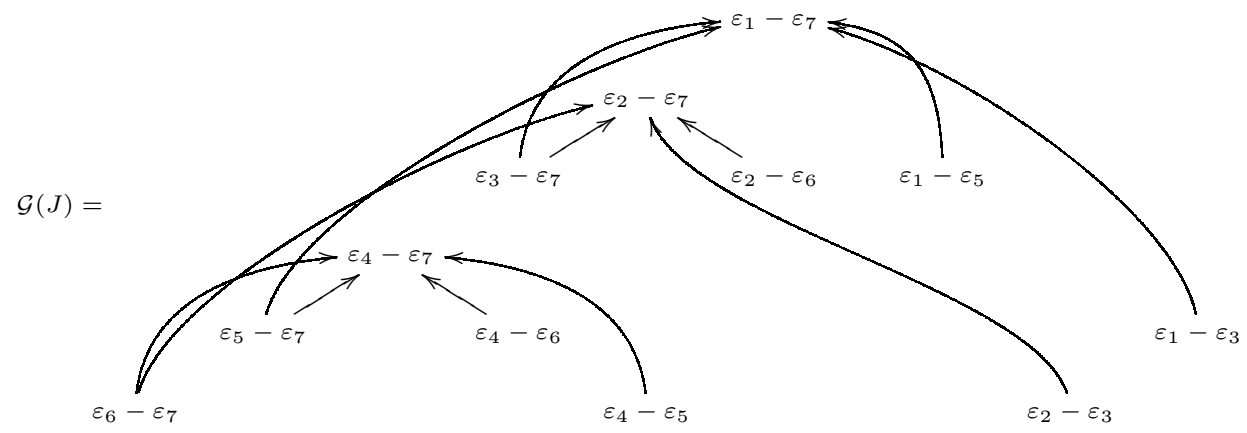

\section{PATtern Group orbits AND CO-ORBITS}

Both superclasses and supercharacters are indexed by two-sided orbits. This section develops a description of these orbits.

4.1. Pattern group orbits. The group $U_{J}$ acts on $\mathfrak{n}_{J}$ by left and right multiplication. Let

$$
O_{\phi}=\left\{U_{J} \times U_{J} \text {-orbits containing } X_{\phi}\right\} .
$$

Of course, multiplying on the right by $x_{\beta}(t)$ where $\beta=(i, j)$ and $t \in \mathbb{F}_{q}$ adds $t$ times column $i$ to column $j$, while adding on the left adds $t$ times row $j$ to row $i$. In the present notation we obtain the following lemma.

Lemma 4.1 (Row and column reducing). Let $\beta \in J, t \in \mathbb{F}_{q}$, and $\phi \in J^{*}$. Then

(a) $X_{\phi} x_{\beta}(t)=X_{\phi^{\prime}}$, where

$$
\phi_{\gamma}^{\prime}=\left\{\begin{array}{ll}
\phi_{\gamma}+t \phi_{\gamma-\beta} & \text { if } \gamma-\beta \in J A N D \beta<\gamma-\beta, \\
\phi_{\gamma} & \text { otherwise, }
\end{array} \quad \gamma \in J,\right.
$$

(b) $x_{\beta}(t) X_{\phi}=X_{\phi^{\prime}}$, where

$$
\phi_{\gamma}^{\prime}= \begin{cases}\phi_{\gamma}+t \phi_{\gamma-\beta} & \text { if } \gamma-\beta \in J A N D \gamma-\beta<\beta, \quad \gamma \in J . \\ \phi_{\gamma} & \text { otherwise, }\end{cases}
$$

Proof. (a) Let $J=A \cup\{\beta\} \cup B$ be such that $A<\beta<B$, and let $\phi_{A}$ and $\phi_{B}$ be the restrictions of $\phi$ to $A$ and $B$, respectively. Then

$$
X_{\phi} x_{\beta}(t)=\left(x_{\phi}-1\right) x_{\beta}(t)=x_{A}\left(\phi_{A}\right) x_{\beta}(\phi(\beta)) x_{B}\left(\phi_{B}\right) x_{\beta}(t)-x_{\beta}(t) .
$$

By relation (3.6),

$$
\begin{aligned}
X_{\phi} x_{\beta}(t) & =x_{A}\left(\phi_{A}\right) x_{\beta}(\phi(\beta)+t)\left[x_{B}\left(\phi_{B}\right), x_{\beta}(t)\right]-x_{\beta}(t) \\
& =x_{A}\left(\phi_{A}\right) x_{\beta}(\phi(\beta)+t)\left[x_{B}\left(\phi_{B}\right), x_{\beta}(t)\right]-1-t X_{\beta} \\
& =x_{A}\left(\phi_{A}\right) x_{\beta}(\phi(\beta))\left[x_{B}\left(\phi_{B}\right), x_{\beta}(t)\right]-1 .
\end{aligned}
$$

By Lemma 3.1 (a), if $\alpha \in B$ and $\alpha+\beta \in J$, then $\alpha+\beta \in B$. By relation (3.5), as $x_{\beta}(t)$ moves left through $x_{B}\left(\phi_{B}\right)$ the only new terms that crop up are of the form $x_{\alpha+\beta}\left(t \phi_{B}(\alpha)\right)$, where $\alpha \in B$ and $\alpha+\beta \in J$. By Lemma 3.1 (b), as $x_{\alpha+\beta}\left(t \phi_{B}(\alpha)\right)$ moves from right to left, it commutes with all other terms until it 
hits $x_{\alpha+\beta}\left(\phi_{B}(\alpha+\beta)\right)$. Thus, every $x_{\gamma}\left(\phi_{B}(\gamma)\right)$ in $B$ such that $\gamma-\beta \in J$ becomes $x_{\gamma}\left(\phi_{B}(\gamma)+t \phi_{B}(\gamma-\beta)\right)$, and all the other terms remain the same.

The proof of $(b)$ is similar.

For the poset version of Lemma 4.1, write

$$
\left(i_{1}, i_{2}, \ldots, i_{r}\right) \in \mathcal{P} \quad \text { if } \quad\left(i_{1}, i_{2}\right),\left(i_{2}, i_{3}\right), \ldots,\left(i_{r-1}, i_{r}\right) \in J
$$

so that $\left(i_{1}, i_{2}, \ldots, i_{r}\right) \in \mathcal{P}$ if $\left(i_{1}, i_{2}, \ldots, i_{r}\right)$ is an $r$-chain in the poset.

Lemma 4.2. Let $x_{\varepsilon_{j}-\varepsilon_{k}}(t) \in U_{J}$ and $X_{\phi} \in \mathfrak{n}_{J}$. Then

(a) $X_{\phi} x_{\varepsilon_{j}-\varepsilon_{k}}(t)=X_{\phi^{\prime}}$, where

$$
\phi_{i l}^{\prime}=\left\{\begin{array}{ll}
\phi_{i l}+t \phi_{i j} & \text { if } l=k A N D(i, j, l) \in \mathcal{P}, \\
\phi_{i l} & \text { otherwise }
\end{array} \quad(i, l) \in \mathcal{P},\right.
$$

(b) $x_{\varepsilon_{j}-\varepsilon_{k}}(t) X_{\phi}=X_{\phi^{\prime}}$, where

$$
\phi_{i l}^{\prime}=\left\{\begin{array}{ll}
\phi_{i l}+t \phi_{k l} & \text { if } i=j A N D(i, k, l) \in \mathcal{P}, \\
\phi_{i l} & \text { otherwise, }
\end{array} \quad(i, l) \in \mathcal{P} .\right.
$$

By iterating Lemma 4.1, we obtain

Theorem 4.1. Let $\phi, \rho \in J^{*}$. Then

(a) $x_{\rho} X_{\phi}=X_{\phi^{\prime}}$, where

$$
\phi_{i l}^{\prime}=\phi_{i l}+\sum_{(i, j, l) \in \mathcal{P}} \rho_{i j} \phi_{j l}
$$

(b) $X_{\phi} x_{\rho}=X_{\phi^{\prime}}$, where

$$
\phi_{i l}^{\prime}=\phi_{i l}+\sum_{(i, j, l) \in \mathcal{P}} \phi_{i j} \rho_{j l} .
$$

Proof. (a) List the roots in $J$ according to the total order, so

$$
\beta_{|J|} \leq \cdots \leq \beta_{2} \leq \beta_{1}
$$

We can apply Lemma 4.1 iteratively:

$$
\left(x_{\beta_{|J|}}\left(\rho\left(\beta_{|J|}\right)\right)\left(\cdots\left(x_{\beta_{2}}\left(\rho\left(\beta_{2}\right)\right)\left(x_{\beta_{1}}\left(\rho\left(\beta_{1}\right)\right) X_{\phi}\right)\right) \cdots\right)\right) .
$$

Induct on $1 \leq r \leq|J|$. By induction, if

$$
X_{\phi^{(r-1)}}=\left(x_{\beta_{r-1}}\left(\rho\left(\beta_{r-1}\right)\right)\left(\cdots\left(x_{\beta_{2}}\left(\rho\left(\beta_{2}\right)\right)\left(x_{\beta_{1}}\left(\rho\left(\beta_{1}\right)\right) X_{\phi}\right)\right) \cdots\right)\right)
$$

then

$$
\phi_{i l}^{(r-1)}=\phi_{i l}+\sum_{\substack{(i, j, l) \in \mathcal{P} \\ \beta_{r}<(i, j)}} \rho_{i j} \phi_{j l} .
$$

Let $X_{\phi^{(r)}}=x_{\beta_{r}}\left(\rho\left(\beta_{r}\right)\right) X_{\phi^{(r-1)}}$. By Lemma 4.1,

$$
\phi_{i l}^{(r)}= \begin{cases}\left(\phi_{i l}^{(r-1)}+\rho_{a b} \phi_{b l}^{(r-1)}\right. & \text { if } a=i \text { and }(a, b, l) \in \mathcal{P}, \\ \phi_{i l}^{(r-1)} & \text { otherwise. }\end{cases}
$$


However, if $a=i$ and $(a, b, l) \in \mathcal{P}$, then by the choice of ordering, the set $\{(b, k, l) \in$ $\left.\mathcal{P} \mid \beta_{r}<(b, k)\right\}$ must be empty (since $\left.a \leq b\right)$. Thus,

$$
\begin{aligned}
\phi_{i l}^{(r)} & = \begin{cases}\phi_{i l}^{(r-1)}+\rho_{a b} \phi_{b l} & \text { if } a=i \text { and }(a, b, l) \in \mathcal{P}, \\
\phi_{i l}^{(r-1)} & \text { otherwise }\end{cases} \\
& =\phi_{i l}+\sum_{\substack{(i, j, l) \in \mathcal{P} \\
\beta_{r} \leq(i, j)}} \rho_{i j} \phi_{j l},
\end{aligned}
$$

as desired.

The proof for (b) is similar.

By first applying (a) and then (b) of the theorem to $X_{\phi}$, we obtain the following corollary.

Corollary 4.1. Let $\phi, \tau, \rho \in J^{*}$. If $X_{\phi^{\prime}}=x_{\tau} X_{\phi} x_{\rho}$, then

$$
\phi_{i l}^{\prime}=\phi_{i l}+\sum_{(i, j, l) \in \mathcal{P}} \tau_{i j} \phi_{j l}+\sum_{(i, k, l) \in \mathcal{P}} \phi_{i k} \rho_{k l}+\sum_{(i, j, k, l) \in \mathcal{P}} \tau_{i j} \phi_{j k} \rho_{k l} .
$$

Define the matrices $M_{\phi}^{L}$ and $M_{\phi}^{R}$ by

$$
\begin{array}{ll}
\left(M_{\phi}^{L}\right)_{(i, l),(j, k)}= \begin{cases}\phi_{k l} & \text { if } i=j,(i, k, l) \in \mathcal{P}, \\
0 & \text { otherwise, }\end{cases} \\
\left(M_{\phi}^{R}\right)_{(i, l),(j, k)}= \begin{cases}\phi_{i j} & \text { if } k=l,(i, j, l) \in \mathcal{P}, \\
0 & \text { otherwise, }\end{cases}
\end{array}
$$

Corollary 4.2. Let $\phi \in J^{*}$. Then

(a) The size of the right orbit containing $X_{\phi}$ is $q^{\operatorname{rank}\left(M_{\phi}^{R}\right)}$.

(b) The size of the left orbit containing $X_{\phi}$ is $q^{\operatorname{rank}\left(M_{\phi}^{L}\right)}$.

Proof. (a) Consider the vector space isomorphism

$$
\begin{aligned}
v: \mathfrak{n}_{J} & \longrightarrow \mathbb{F}_{q}^{|J|} \\
X_{\rho} & \mapsto v_{\rho},
\end{aligned} \quad \text { where } \quad\left(v_{\rho}\right)_{(i, j)}=\rho_{i j} .
$$

By Theorem 4.1 the equation

$$
X_{\phi} x_{\rho}=X_{\phi}+X_{\phi} X_{\rho}
$$

becomes

$$
v\left(X_{\phi} x_{\rho}\right)=v_{\phi}+M_{\phi}^{R} v_{\rho} .
$$

Thus, the right orbit containing $X_{\phi}$ has the same size as

$$
\left|\left\{M_{\phi}^{R} v \mid v \in \mathbb{F}_{q}^{|J|}\right\}\right|=q^{\operatorname{rank}\left(M_{\phi}^{R}\right)} .
$$

The proof of (b) is similar.

Cautionary example. For the full upper-triangular group, much of the charactertheoretic information depends only on the "shape" or the support of the particular 
$\phi$ or $\eta$ in $J^{*}$. 8 . The following example shows that this does not hold for general pattern groups (at least in the obvious way). Consider $q \geq 3$ and

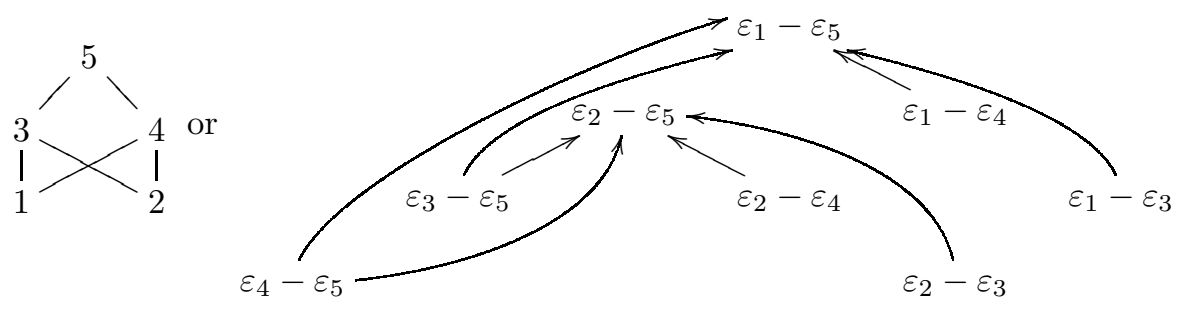

The group $U_{J}$ is then the set of matrices of the form

$$
\left(\begin{array}{lllll}
1 & 0 & * & * & * \\
0 & 1 & * & * & * \\
0 & 0 & 1 & 0 & * \\
0 & 0 & 0 & 1 & * \\
0 & 0 & 0 & 0 & 1
\end{array}\right)
$$

The matrices

$$
x_{1}=\left(\begin{array}{ccccc}
1 & 0 & 1 & 1 & 0 \\
0 & 1 & 1 & 1 & 0 \\
0 & 0 & 1 & 0 & 0 \\
0 & 0 & 0 & 1 & 0 \\
0 & 0 & 0 & 0 & 1
\end{array}\right) \quad \text { and } \quad x_{2}=\left(\begin{array}{ccccc}
1 & 0 & 2 & 1 & 0 \\
0 & 1 & 1 & 1 & 0 \\
0 & 0 & 1 & 0 & 0 \\
0 & 0 & 0 & 1 & 0 \\
0 & 0 & 0 & 0 & 1
\end{array}\right)
$$

have two-sided orbits given by

$$
\left\{\left(\begin{array}{ccccc}
1 & 0 & 1 & 1 & a \\
0 & 1 & 1 & 1 & a \\
0 & 0 & 1 & 0 & 0 \\
0 & 0 & 0 & 1 & 0 \\
0 & 0 & 0 & 0 & 1
\end{array}\right) \mid a \in \mathbb{F}_{q}\right\} \quad \text { and }\left\{\left(\begin{array}{ccccc}
1 & 0 & 2 & 1 & a \\
0 & 1 & 1 & 1 & b \\
0 & 0 & 1 & 0 & 0 \\
0 & 0 & 0 & 1 & 0 \\
0 & 0 & 0 & 0 & 1
\end{array}\right) \mid a, b \in \mathbb{F}_{q}\right\},
$$

respectively, so although $x_{1}$ and $x_{2}$ have the same "shape," they have different size superclasses.

4.2. Pattern group co-orbits. The group $U_{J}$ acts on the dual space $\mathfrak{n}^{*}$ on the left and right by

$$
x \lambda\left(X_{\phi}\right) y=\lambda\left(x^{-1} X_{\phi} y^{-1}\right), \quad \text { for } x, y \in U_{J}, \lambda \in \mathfrak{n}^{*} .
$$

For $\eta \in J^{*}$, let

$$
O^{\eta}=\left\{U_{J} \times U_{J} \text {-orbits containing } \lambda_{\eta}\right\} .
$$

Lemma 4.3 (Dual row and column reducing). Let $\beta \in J, t \in \mathbb{F}_{q}$, and $\eta: J \rightarrow \mathbb{F}_{q}$. Then

(a) $\lambda_{\eta} x_{\beta}(-t)=\lambda_{\eta^{\prime}}$, where

$$
\eta_{\alpha}^{\prime}=\left\{\begin{array}{ll}
\eta_{\alpha}+t \eta_{\alpha+\beta} & \text { if } \alpha+\beta \in J \text { AND } \beta<\alpha+\beta, \\
\eta_{\alpha} & \text { otherwise, }
\end{array} \quad \alpha \in J,\right.
$$

(b) $x_{\beta}(-t) \lambda_{\eta}=\lambda_{\eta^{\prime}}$, where

$$
\eta_{\alpha}^{\prime}=\left\{\begin{array}{ll}
\eta_{\alpha}+t \eta_{\alpha+\beta} & \text { if } \alpha+\beta \in J \text { AND } \alpha+\beta<\beta, \\
\eta_{\alpha} & \text { otherwise, }
\end{array} \quad \alpha \in J .\right.
$$


Proof. (a) If $\alpha \leq \beta$ OR $\alpha+\beta \notin J$, then

$$
\lambda_{\eta}\left(X_{\alpha}\right) x_{\beta}(-t)=\lambda_{\eta}\left(x_{\alpha}(1) x_{\beta}(t)-x_{\beta}(t)\right)=\lambda_{\eta}\left(X_{\alpha}\right) .
$$

If $\beta<\alpha$ AND $\alpha+\beta \in J$, then

$$
\begin{aligned}
\lambda_{\eta}\left(X_{\alpha}\right) x_{\beta}(-t) & =\sum_{\gamma \in J} \eta_{\gamma} \lambda_{\gamma}\left(X_{\alpha} x_{\beta}(t)\right) \\
& =\sum_{\gamma \in J} \eta_{\gamma} \lambda_{\gamma}\left(t X_{\alpha+\beta}+X_{\alpha}\right) \\
& =t \eta_{\alpha+\beta} \lambda_{\alpha+\beta}\left(X_{\alpha+\beta}\right)+\eta_{\alpha} \lambda_{\alpha}\left(X_{\alpha}\right) \\
& =\left(\lambda_{\eta}+t \eta_{\alpha+\beta} \lambda_{\alpha}\right)\left(X_{\alpha}\right) .
\end{aligned}
$$

Thus,

$$
\lambda_{\eta} x_{\beta}(-t)=\lambda_{\eta^{\prime}}, \quad \text { where } \eta_{\alpha}^{\prime}= \begin{cases}\eta_{\alpha}+t \eta_{\alpha+\beta} & \text { if } \alpha+\beta \in J \text { AND } \beta<\alpha+\beta, \\ \eta_{\alpha} & \text { otherwise. }\end{cases}
$$

The proof of (b) is similar.

The poset version of Lemma 4.3 is

Lemma 4.4. Let $x_{\varepsilon_{i}-\varepsilon_{l}}(t) \in U_{J}, t \in \mathbb{F}_{q}$, and $\eta \in J^{*}$. Then

(a) $x_{\varepsilon_{i}-\varepsilon_{l}}(-t) \lambda_{\eta}=\lambda_{\eta^{\prime}}$, where

$$
\eta_{j k}^{\prime}= \begin{cases}\eta_{j k}+t \eta_{i k} & \text { if } l=j \text { AND }(i, j, k) \in \mathcal{P}, \quad(j, k) \in \mathcal{P}, \\ \eta_{j k} & \text { otherwise, }\end{cases}
$$

(b) $\lambda_{\eta} x_{\varepsilon_{i}-\varepsilon_{l}}(-t)=\lambda_{\eta^{\prime}}$, where

$$
\eta_{j k}^{\prime}= \begin{cases}\eta_{j k}+t \eta_{j l} & \text { if } i=k \text { AND }(j, k, l) \in \mathcal{P}, \quad(j, k) \in \mathcal{P} . \\ \eta_{j k} & \text { otherwise, }\end{cases}
$$

We have a result similar to Theorem 4.1, but in this case we act by $x_{\rho}^{-1}$.

Theorem 4.2. Let $\eta, \rho \in J^{*}$. Then

(a) $x_{\rho}^{-1} \lambda_{\eta}=\lambda_{\eta^{\prime}}$, where

$$
\eta_{j k}^{\prime}=\eta_{j k}+\sum_{(i, j, k) \in \mathcal{P}} \rho_{i j} \eta_{i k}
$$

(b) $\lambda_{\eta} x_{\rho}^{-1}=\lambda_{\eta^{\prime}}$, where

$$
\eta_{j k}^{\prime}=\eta_{j k}+\sum_{(j, k, l) \in \mathcal{P}} \rho_{k l} \eta_{j l} .
$$

Proof. The proof is the same as for Theorem 4.1, with the reversed ordering.

Combine (a) and (b) from Theorem 4.2 to obtain

Corollary 4.3. Let $\eta, \tau, \rho \in J^{*}$. If $\lambda_{\eta^{\prime}}=x_{\tau}^{-1} \lambda_{\eta} x_{\rho}^{-1}$, then

$$
\eta_{j k}^{\prime}=\eta_{j k}+\sum_{(i, j, k) \in \mathcal{P}} \tau_{i j} \eta_{i k}+\sum_{(j, k, l) \in \mathcal{P}} \eta_{j l} \rho_{k l}+\sum_{(i, j, k, l) \in \mathcal{P}} \tau_{i j} \eta_{i l} \rho_{k l} .
$$


Define the $|J| \times|J|$ matrices $M_{L}^{\eta}$ and $M_{R}^{\eta}$ by

$$
\begin{array}{ll}
\left(M_{L}^{\eta}\right)_{(j, k),(i, l)}= \begin{cases}\eta_{i k} & \text { if } l=j,(i, j, k) \in \mathcal{P}, \\
0 & \text { otherwise, }\end{cases} \\
\left(M_{R}^{\eta}\right)_{(j, k),(i, l)}= \begin{cases}\eta_{j l} & \text { if } k=i,(j, k, l) \in \mathcal{P}, \\
0 & \text { otherwise, }\end{cases}
\end{array}
$$

Note that

$$
\left(M_{L}^{\eta}\right)_{(j, k),(i, j)}=\eta_{i k} \quad \text { if and only if } \quad\left(M_{R}^{\eta}\right)_{(i, j),(j, k)}=\eta_{i k} .
$$

Thus, $\operatorname{rank}\left(M_{L}^{\eta}\right)=\operatorname{rank}\left(M_{R}^{\eta}\right)$. Define the corank of $\eta$ to be

$$
\operatorname{corank}(\eta)=\operatorname{rank}\left(M_{L}^{\eta}\right)=\operatorname{rank}\left(M_{R}^{\eta}\right) .
$$

Corollary 4.4. Let $\eta \in J^{*}$. Then

(a) The size of the right orbit containing $\lambda_{\eta}$ is $q^{\operatorname{corank}(\eta)}$.

(b) The size of the left orbit containing $\lambda_{\eta}$ is $q^{\operatorname{corank}(\eta)}$.

Cautionary example. Two co-orbits with the same support shape can also have different co-orbit sizes (cf. cautionary example in Section 4.1). A similar argument to the one in Section 4.1 gives that

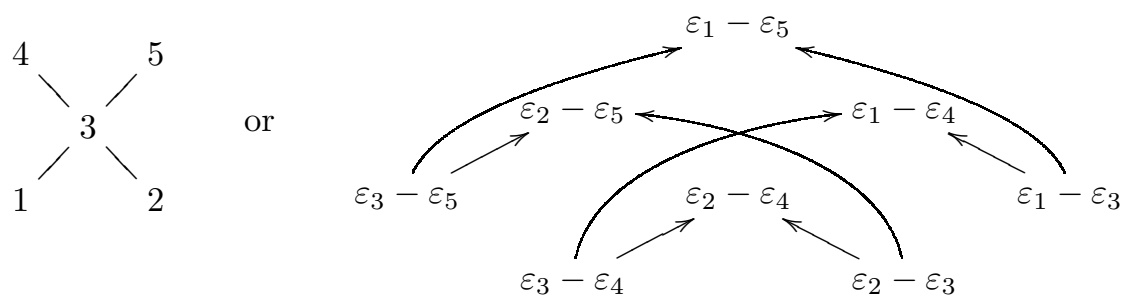

has co-orbits whose sizes depend on more than their shape. Furthermore, while the number of orbits equals the number of co-orbits [10, Lemma 4.1], the sizes of the two-sided orbits for an orbit or co-orbit indexed by the same symbol $\eta$ can differ (in fact, they usually will).

\section{A PATtern group SUPERChaRACTER Formula}

This section states and proves our main theorem, a formula for a supercharacter on a superclass. Following this, two examples (Heisenberg and $U_{n}\left(\mathbb{F}_{q}\right)$ ) show how the formula can be used.

The superclass corresponding to $\phi \in J^{*}$ is

$$
\left\{x_{\rho} \mid \rho \in O_{\phi}\right\}
$$

and is a union of conjugacy classes in $U_{J}$.

Fix a nontrivial homomorphism $\theta: \mathbb{F}_{q}^{+} \rightarrow \mathbb{C}^{\times}$. For $\eta \in J^{*}$, let $\chi^{\eta}: U_{J} \rightarrow \mathbb{C}$ be the map given by

$$
\begin{aligned}
\chi^{\eta}\left(x_{\phi}\right) & =\frac{q^{\operatorname{corank}(\eta)}}{\left|O^{\eta}\right|} \sum_{\mu \in O^{\eta}} \theta\left(\lambda_{\mu}\left(X_{\phi}\right)\right) \\
& \left.=\frac{q^{\operatorname{corank}(\eta)}}{\left|O_{\phi}\right|} \sum_{\rho \in O_{\phi}} \overline{\theta\left(\lambda_{\eta}\left(X_{\rho}\right)\right.}\right) .
\end{aligned}
$$


By [10, Theorems 5.6 and 5.8], these maps are the supercharacters of $U_{J}$. They are constant on superclasses and are orthogonal under the usual inner product with the relation [10, Lemma 5.9]

$$
\left\langle\chi^{\eta}, \chi^{\mu}\right\rangle=\delta_{\eta \mu} \frac{q^{2 \operatorname{corank}(\eta)}}{\left|O^{\eta}\right|} .
$$

To state the main theorem, we need one final piece of notation. For $\phi, \eta \in J^{*}$, let $M_{\phi}^{\eta}$ be the $|J| \times|J|$ matrix given by

$$
\left(M_{\phi}^{\eta}\right)_{(i, j)(k, l)}= \begin{cases}\phi_{j k} \eta_{i l} & \text { if }(i, j, k, l) \in \mathcal{P} \\ 0 & \text { otherwise. }\end{cases}
$$

Let $a_{\phi}^{\eta} \in \mathbb{F}_{q}^{|J|}$ and $b_{\phi}^{\eta} \in \mathbb{F}_{q}^{|J|}$ be given by

$$
\begin{aligned}
& \left(a_{\phi}^{\eta}\right)_{(i, j)}=\sum_{(i, j, k) \in \mathcal{P}} \phi_{j k} \eta_{i k}, \\
& \left(b_{\phi}^{\eta}\right)_{(j, k)}=\sum_{(i, j, k) \in \mathcal{P}} \phi_{i j} \eta_{i k} .
\end{aligned}
$$

The functional $\phi$ meshes with $\eta$ if

(1) a solution to the equation $M_{\phi}^{\eta} x=-a_{\phi}^{\eta}$ exists,

(2) $b_{\phi}^{\eta}$ is perpendicular to the nullspace of $M_{\phi}^{\eta}$ (with respect to the usual dot product).

The following theorem gives a formula for a supercharacter on a superclass.

Theorem 5.1. Let $\phi, \eta \in J^{*}$. Let $M_{\phi}^{\eta}, a_{\phi}^{\eta}$, and $b_{\phi}^{\eta}$ be as in (5.1), (5.2), and (5.3). Then

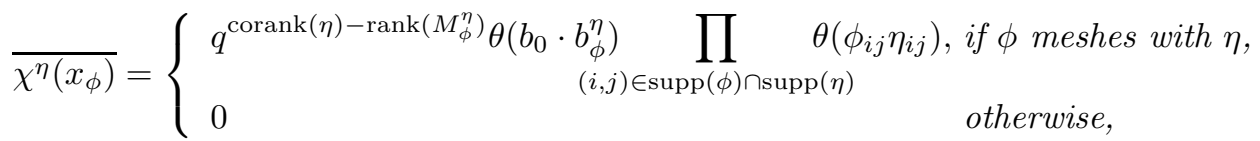

where $b_{0} \in \mathbb{F}_{q}^{|J|}$ satisfies $M_{\phi}^{\eta} b_{0}=-a_{\phi}^{\eta}$.

Proof. Let $\mathbb{F}_{J}=\mathbb{F}_{q}^{|J|}$. For $a, b \in \mathbb{F}_{J}$, let $X_{a b}=X_{\phi^{\prime}}$, where

$$
\phi_{i l}^{\prime}=\phi_{i l}+\sum_{(i, j, l) \in \mathcal{P}} a_{i j} \phi_{j l}+\sum_{(i, k, l) \in \mathcal{P}} \phi_{i k} b_{k l}+\sum_{(i, j, k, l) \in \mathcal{P}} a_{i j} \phi_{j k} b_{k l} .
$$

Since $\left|O_{\phi}\right|$ divides $q^{2|J|}$, we can overcount to get

$$
\overline{\chi^{\eta}\left(x_{\phi}\right)}=\frac{q^{\operatorname{corank}(\eta)}}{\left|O_{\phi}\right|} \sum_{\rho \in \kappa_{\phi}} \theta\left(\lambda_{\eta}\left(X_{\rho}\right)\right)=\frac{q^{\operatorname{corank}(\eta)}}{q^{2|J|}} \sum_{a, b \in \mathbb{F}_{J}} \theta\left(\lambda_{\eta}\left(X_{a b}\right)\right) .
$$


By the definitions of $\lambda_{\eta}$ and $X_{a b}$,

$$
\begin{aligned}
& \overline{\chi^{\eta}\left(x_{\phi}\right)}=\frac{q^{\operatorname{corank}(\eta)}}{q^{2|J|}} \sum_{a, b \in \mathbb{F}_{J}} \theta\left(\sum _ { ( i , l ) \in J } \eta _ { i l } \left(\phi_{i l}+\sum_{(i, j, l) \in \mathcal{P}} a_{i j} \phi_{j l}+\phi_{i j} b_{j l}\right.\right. \\
& \left.\left.+\sum_{(i, j, k, l) \in \mathcal{P}} a_{i j} \phi_{j k} b_{k l}\right)\right) \\
& =\frac{q^{\operatorname{corank}(\eta)} \theta_{\phi \eta}}{q^{2|J|}} \sum_{a, b \in \mathbb{F}_{J}} \theta\left(\sum _ { ( i , l ) \in J } \eta _ { i l } \left(\sum_{(i, j, l) \in \mathcal{P}} a_{i j} \phi_{j l}+\phi_{i j} b_{j l}\right.\right. \\
& \left.\left.+\sum_{(i, j, k, l) \in \mathcal{P}} a_{i j} \phi_{j k} b_{k l}\right)\right),
\end{aligned}
$$

where the second equality comes by collecting all summands that do not depend on $a$ and $b$,

$$
\theta_{\phi \eta}=\theta\left(\sum_{(i, l) \in J} \eta_{i l} \phi_{i l}\right)=\prod_{(i, j) \in \operatorname{supp}(\phi) \cap \operatorname{supp}(\eta)} \theta\left(\eta_{i l} \phi_{i l}\right) .
$$

Collecting summands that contain $a_{i j}$ for all $(i, j) \in J$,

$$
\begin{array}{r}
\sum_{a, b \in \mathbb{F}_{J}} \theta\left(\sum_{(i, l) \in J} \eta_{i l}\left(\sum_{(i, j, l) \in \mathcal{P}} a_{i j} \phi_{j l}+\phi_{i j} b_{j l}+\sum_{(i, j, k, l) \in \mathcal{P}} a_{i j} \phi_{j k} b_{k l}\right)\right) \\
=\sum_{a, b \in \mathbb{F}_{J}} \theta\left(\sum_{(i, j) \in J} a_{i j}\left(\sum_{(i, j, k) \in \mathcal{P}} \phi_{j k} \eta_{i k}+\sum_{(i, j, k, l) \in \mathcal{P}} \phi_{j k} \eta_{i l} b_{k l}\right)\right. \\
\left.+\sum_{(j, k, l) \in \mathcal{P}} \phi_{j k} \eta_{j l} b_{k l}\right) \\
=\sum_{a, b \in \mathbb{F}_{J}} \prod_{(i, j) \in J} \theta\left(a_{i j}\left(\sum_{(i, j, k) \in \mathcal{P}} \phi_{j k} \eta_{i k}+\sum_{(i, j, k, l) \in \mathcal{P}} \phi_{j k} \eta_{i l} b_{k l}\right)\right. \\
\left.+\sum_{(j, k, l) \in \mathcal{P}} \phi_{j k} \eta_{j l} b_{k l}\right) .
\end{array}
$$

Note that if for any $(i, j) \in J$,

$$
\sum_{(i, j, k) \in \mathcal{P}} \phi_{j k} \eta_{i k}+\sum_{(i, j, k, l) \in \mathcal{P}} \phi_{j k} \eta_{i l} b_{k l} \neq 0,
$$

then as we sum over all possible values of $a_{i j} \in \mathbb{F}_{q}$, we sum over all roots of unity and

$$
\sum_{a_{i j} \in \mathbb{F}_{q}} \theta\left(a_{i j}\left(\sum_{(i, j, k) \in \mathcal{P}} \phi_{j k} \eta_{i k}+\sum_{(i, j, k, l) \in \mathcal{P}} \phi_{j k} \eta_{i l} b_{k l}\right)+\sum_{(j, k, l) \in \mathcal{P}} \phi_{j k} \eta_{j l} b_{k l}\right)=0 .
$$


If

then the equation

$$
\mathcal{S}=\left\{b \in \mathbb{F}_{J} \mid M_{\phi}^{\eta} b=-a_{\phi}^{\eta}\right\}
$$

$$
\sum_{(i, j, k) \in \mathcal{P}} \phi_{j k} \eta_{i k}+\sum_{(i, j, k, l) \in \mathcal{P}} \phi_{j k} \eta_{i l} b_{k l}=\left(a_{\phi}^{\eta}\right)_{i j}+\left(M_{\phi}^{\eta} b\right)_{i j}, \quad \text { for }(i, j) \in J
$$

implies

$\overline{\chi^{\eta}\left(x_{\phi}\right)}=\frac{q^{\operatorname{corank}(\eta)} \theta_{\phi \eta}}{q^{2|J|}} \sum_{a \in \mathbb{F}_{J}, b \in \mathcal{S}} \theta\left(\sum_{(j, k, l) \in \mathcal{P}} \phi_{j k} \eta_{j l} b_{k l}\right)=\frac{q^{\operatorname{corank}(\eta)} \theta_{\phi \eta}}{q^{|J|}} \sum_{b \in \mathcal{S}} \theta\left(b_{\phi}^{\eta} \cdot b\right)$.

Fix an element $b_{0} \in \mathcal{S}$. Since every other vector in $\mathcal{S}$ is of the form $b_{0}+b^{\prime}$, where $b^{\prime}$ is in the nullspace of $M_{\phi}^{\eta}$, we have

$$
\overline{\chi^{\eta}\left(x_{\phi}\right)}=\frac{q^{\operatorname{corank}(\eta)} \theta_{\phi \eta} \theta\left(b_{0} \cdot b_{\phi}^{\eta}\right)}{q^{|J|}} \sum_{b^{\prime} \in \operatorname{Null}\left(M_{\phi}^{\eta}\right)} \theta\left(b^{\prime} \cdot b_{\phi}^{\eta}\right) .
$$

Let $\left\{b_{1}^{\prime}, b_{2}^{\prime}, \ldots, b_{r}^{\prime}\right\}$ be a basis for $\operatorname{Null}\left(M_{\phi}^{\eta}\right)$. Then

$$
\sum_{b^{\prime} \in \operatorname{Null}\left(M_{\phi}^{\eta}\right)} \theta\left(b^{\prime} \cdot b_{\phi}^{\eta}\right)=\sum_{t \in \mathbb{F}_{q}^{r}} \prod_{i=1}^{r} \theta\left(t_{i} b_{i}^{\prime} \cdot b_{\phi}^{\eta}\right)=\prod_{i=1}^{r} \sum_{t_{i} \in \mathbb{F}_{q}} \theta\left(t_{i} b_{i}^{\prime} \cdot b_{\phi}^{\eta}\right) .
$$

Thus, if any $b_{i}^{\prime}$ is not orthogonal to $b_{\phi}^{\eta}$, then the product is zero. Thus, if $\phi$ meshes with $\eta$, we get

$$
\begin{aligned}
\overline{\chi^{\eta}\left(x_{\phi}\right)} & =\frac{q^{\operatorname{corank}(\eta)} \theta_{\phi \eta} \theta\left(b_{0} \cdot b_{\phi}^{\eta}\right)}{q^{|J|}}\left|\operatorname{Null}\left(M_{\phi}^{\eta}\right)\right|=\frac{q^{\operatorname{corank}(\eta)} \theta_{\phi \eta} \theta\left(b_{0} \cdot b_{\phi}^{\eta}\right)}{q^{|J|}} q^{|J|-\operatorname{rank}\left(M_{\phi}^{\eta}\right)} \\
& =q^{\operatorname{corank}(\eta)-\operatorname{rank}\left(M_{\phi}^{\eta}\right)} \theta_{\phi \eta} \theta\left(b_{0} \cdot b_{\phi}^{\eta}\right),
\end{aligned}
$$

as desired.

Remarks. (1) In general, the matrices $M_{\phi}^{\eta}, M_{\phi}^{R}, M_{R}^{\eta}, M_{\phi}^{L}$, and $M_{L}^{\eta}$ have many zero rows and columns. If one is careful, one can significantly reduce the dimensions of these matrices using the structure of the corresponding posets, but for expository purposes we omitted such "simplifications."

(2) We have avoided choosing orbit and co-orbit representatives in stating Theorem 5.1. In concrete cases, there appear to be natural choices (often with minimal support). For example, the Heisenberg group, below, orbits and co-orbits can be identified with cosets of the center. With $U_{n}\left(\mathbb{F}_{q}\right)$, below, orbits and co-orbits can be identified with labeled set partitions as described in $[1,2,3,4,8,17$.

\subsection{Examples.}

Example 1: Heisenberg group. Let $H_{n}$ be the group of order $q^{2 n-3}$ represented as $n \times n$ upper-triangular matrices with ones on the diagonal, entries in $\mathbb{F}_{q}$, and nonzero entries allowed only in the top row or last column. That is, let $H_{n}$ be the pattern group $U_{J}$ with

$$
J=\{(n-1, n),(n-2, n), \ldots,(1, n),(1, n-1), \ldots,(1,2)\},
$$


and corresponding poset

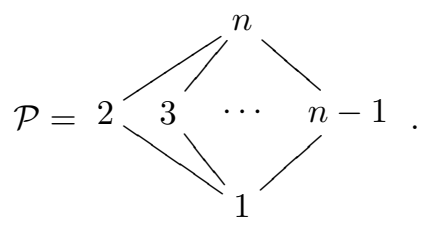

The structure and character theory of $H_{n}$ is well known. The center

$$
Z\left(H_{n}\right)=\text { the commutator of } H_{n}=\left\{x_{1 n}(t) \mid t \in \mathbb{F}_{q}\right\} \text {. }
$$

Thus, the conjugacy classes of $H_{n}$ consist of $Z\left(H_{n}\right)$ together with the $\left(q^{2 n-4}-1\right)$ other cosets of $Z\left(H_{n}\right)$. There are $q^{2 n-4}$ linear characters and $(q-1)$ irreducible characters of degree $q^{n-2}$.

Consider the supercharacter theory of $H_{n}$. From Corollary 4.1 (or direct computation), $x_{\tau} X_{\phi} x_{\rho}=X_{\phi^{\prime}}$ with $\phi_{i l}^{\prime}=\phi_{i l}$ unless $(i, l)=(1, n)$ and

$$
\phi_{1 n}^{\prime}=\phi_{1 n}+\sum_{j=2}^{n-1}\left(\tau_{1 j} \phi_{1 n}+\phi_{1 n} \rho_{j n}\right) .
$$

It follows that each superclass is in fact a single conjugacy class. From this, the supercharacters are each irreducible. These facts also follow from Corollary [5.1. below.

The character formula of Theorem 5.1 is simple for this example. Vectors and matrices $M, a$, and $b$ can be indexed by $J$ in the linear order of (5.4) above. Note that for any $\phi, \eta \in J^{*}$ the matrix $M_{\phi}^{\eta}=0$ since there is never $(i, j, k, l) \in \mathcal{P}$. Note that

$$
\left(a_{\phi}^{\eta}\right)_{i j}=\left\{\begin{array}{ll}
\eta_{1 n} \phi_{j n} & \text { if }(i, j)=(1, j), \\
0 & \text { otherwise, }
\end{array} \quad \text { and } \quad\left(b_{\phi}^{\eta}\right)_{j k}= \begin{cases}\eta_{1 n} \phi_{1 j} & \text { if }(j, k)=(j, n), \\
0 & \text { otherwise }\end{cases}\right.
$$

Now $\phi$ and $\eta$ mesh if $a_{\phi}^{\eta}=b_{\phi}^{\eta}=0$. Thus, $\phi$ and $\eta$ mesh if and only if either

(a) $\eta_{1 n}=0$, or

(b) $\phi_{i j}=0$ for all positions $(i, j) \neq(1, n)$.

In both cases, $\operatorname{rank}\left(M_{\phi}^{\eta}\right)=0$. By Corollary 4.3,

$$
\operatorname{corank}(\eta)=\operatorname{rank}\left(M_{L}^{\eta}\right)= \begin{cases}0 & \text { if } \eta_{1 n}=0 \\ n-2 & \text { otherwise }\end{cases}
$$

The vector $b$ in Theorem 5.1 can be taken to be $0\left(M_{\phi}^{\eta} b=-a_{\phi}^{\eta}\right)$, so

$$
\overline{\chi^{\eta}\left(x_{\phi}\right)}= \begin{cases}\prod_{(i, j) \in \mathcal{P}} \theta\left(\phi_{i j} \eta_{i j}\right) & \text { if } \eta_{1 n}=0 \\ q^{n-2} \theta\left(\phi_{1 n} \eta_{1 n}\right) & \text { if } \eta_{1 n} \neq 0, x_{\phi} \in Z\left(H_{n}\right) \\ 0 & \text { otherwise. }\end{cases}
$$

Note that it is natural to choose orbit representatives for cosets of $Z\left(H_{n}\right)$ with $\eta_{1 n}=0$. Then (5.5) gives the usual formula for the irreducible characters of $H_{n}$.

Example 2: $U_{n}\left(\mathbb{F}_{q}\right)$. For the full group of upper-triangular matrices, $U_{n}\left(\mathbb{F}_{q}\right)$, we may reduce the character formula to the one found in [8]. Let $J=R^{+}$. In this case, we may choose our orbit representatives $\phi \in J^{*}$ so that

$$
\phi_{j k} \neq 0 \quad \text { implies } \quad \phi_{j l}=0=\phi_{i k}, \quad \text { for all } j<l \neq k \text { and } j \neq i<k \text {. }
$$


Similarly, choose the co-orbit representatives $\eta \in J^{*}$ such that

$$
\eta_{j k} \neq 0 \quad \text { implies } \quad \eta_{j l}=0=\eta_{i k}, \quad \text { for all } j<l \neq k \text { and } j \neq i<k \text {. }
$$

That is, in either case we are permitted at most one nonzero entry for each row and column of $X_{\phi}, X_{\eta} \in \mathfrak{n}$. For such choices we may now compute $\chi^{\eta}\left(x_{\phi}\right)$.

By our choice of representatives $\eta$ and $\phi$, the matrix $M_{\phi}^{\eta}$ has at most one nonzero entry in every row and column. Suppose $\left(M_{\phi}^{\eta}\right)_{(i j)(k l)} \neq 0$ so that $(i, j, k, l) \in \mathcal{P}$, $\phi_{j k} \neq 0$, and $\eta_{i l} \neq 0$. Since

$$
\left(a_{\phi}^{\eta}\right)_{(i j)}=\sum_{(i, j, k)} \phi_{j k} \eta_{i k},
$$

our choice of representatives implies that in each summand either $\phi_{j k}=0$ or $\eta_{i k}=0$. Thus, for every row of $M_{\phi}^{\eta}$ that has a nonzero row, the corresponding entry in $a_{\phi}^{\eta}$ is zero, and a solution to the matrix equation

$$
M_{\phi}^{\eta} b_{0}=-a_{\phi}^{\eta}
$$

exists (and can be $b_{0}=0$ ) if and only if $a_{\phi}^{\eta}=0$. Note that $a_{\phi}^{\eta}=0$ implies that if $(i, j, k) \in \mathcal{P}$, then either $\phi_{j k}=0$ or $\eta_{i k}=0$. In other words, any entry in $\operatorname{supp}(\eta)$ that shares a column with an entry of $\operatorname{supp}(\phi)$ must be below that entry.

The nullspace $\operatorname{Null}\left(M_{\phi}^{\eta}\right)$ of $M_{\phi}^{\eta}$ has as a basis

$$
\operatorname{Null}\left(M_{\phi}^{\eta}\right)=\mathbb{C}-\operatorname{span}\left\{e_{(k, l)} \mid \text { the }(k, l) \text { column of } M_{\phi}^{\eta} \text { is zero }\right\},
$$

where $e_{(k, l)}$ is the vector with one in the $(k, l)$-slot and zeroes elsewhere. Thus, for $b_{\phi}^{\eta}$ to be perpendicular to $\operatorname{Null}\left(M_{\phi}^{\eta}\right)$ it suffices that $\left(b_{\phi}^{\eta}\right)_{(k, l)}=0$ for every column $(k, l)$ of $M_{\phi}^{\eta}$ which has no nonzero entry. Since

$$
\left(b_{\phi}^{\eta}\right)_{k, l}=\sum_{(i, k, l)} \phi_{i k} \eta_{i l},
$$

this condition implies that for all $(i, k, l) \in \mathcal{P}$, either $\phi_{i k}=0$ or $\eta_{i l}=0$. In other words, any entry in $\operatorname{supp}(\eta)$ that shares a row with an entry of $\operatorname{supp}(\phi)$ must be to the left of that entry.

We may conclude $\chi^{\eta}\left(x_{\phi}\right)$ is nonzero $(\eta$ meshes with $\phi)$ if and only if the following two conditions hold:

(a) $\phi_{i j} \neq 0$ and $\eta_{i l} \neq 0$ implies $j \geq l$,

(b) $\phi_{j k} \neq 0$ and $\eta_{i k} \neq 0$ implies $i \leq j$.

This gives us a combinatorial interpretation of everything in the character formula except for the power of $q$.

By the choice of $\eta$, the matrix $M_{R}^{\eta}$ has at most one nonzero entry in every row and column. Thus, the $\operatorname{corank}(\eta)=\operatorname{rank}\left(M_{R}^{\eta}\right)$ is the number of nonzero entries in $M_{R}^{\eta}$, which is

$$
\left|\left\{(i, j, k) \in \mathcal{P} \quad \mid \eta_{i k} \neq 0\right\}\right|=\sum_{\substack{(i, k) \in \mathcal{P} \\ \eta_{i k} \neq 0}} k-i-1 .
$$

In terms of matrices, $k-i-1$ is the number of entries below the $(i, k)$ entry and above the diagonal.

The rank of $M_{\phi}^{\eta}$ is also the number of nonzero entries in $M_{\phi}^{\eta}$, so

$$
\operatorname{rank}\left(M_{\phi}^{\eta}\right)=\left|\left\{(i, j, k, l) \in \mathcal{P} \mid \phi_{j k} \neq 0, \eta_{i l} \neq 0\right\}\right| .
$$


In terms of matrices,

$$
\begin{array}{r}
\operatorname{corank}(\eta)-\operatorname{rank}\left(M_{\phi}^{\eta}\right)=\sum_{(i, l) \in \operatorname{supp}(\eta)}\left(\begin{array}{c}
\text { Number of entries } \\
\text { below }(i, l) \text { and } \\
\text { above the diagonal }
\end{array}\right) \\
-\left(\begin{array}{c}
\text { Number of }(j, k) \in \operatorname{supp}(\phi) \\
\text { such that }(j, k) \text { is } \\
\text { strictly SouthWest of }(i, l)
\end{array}\right) .
\end{array}
$$

In the language of posets, if $\mathcal{P}_{i j}$ is the interval in $\mathcal{P}$ from $i$ to $j$, then

$$
\operatorname{corank}(\eta)-\operatorname{rank}\left(M_{\phi}^{\eta}\right)=\sum_{(i, l) \in \operatorname{supp}(\eta)}\left|\mathcal{P}_{i+1, l-1}\right|-\left|\operatorname{supp}(\phi) \cap \mathcal{P}_{i+1, l-1}\right|,
$$

SO

$$
\begin{aligned}
& \overline{\chi^{\eta}\left(x_{\phi}\right)} \\
& = \begin{cases}\prod_{(i, l) \in \operatorname{supp}(\eta)} q^{\left|\mathcal{P}_{i+1, l-1}\right|-\left|\operatorname{supp}(\phi) \cap \mathcal{P}_{i+1, l-1}\right|} \theta\left(\eta_{i l} \phi_{i l}\right) & \text { if } \begin{array}{c}
\phi_{i j} \neq 0, \eta_{i l} \neq 0 \text { implies } j \geq l, \\
\phi_{j k} \neq 0, \eta_{i k} \neq 0 \text { implies } i \leq j,
\end{array} \\
0 & \text { otherwise. }\end{cases}
\end{aligned}
$$

Example 3: No 4-chains. Let $\mathcal{P}$ be any poset that contains no sequence of elements $i<j<k<l$, and for expository purposes assume that every element is contained in some 3 -chain. Let

$$
\begin{aligned}
\mathcal{T} & =\{k \in \mathcal{P} \quad \mid \quad(i, j, k) \in \mathcal{P} \text { for some } i, j \in \mathcal{P}\}, \\
\mathcal{M} & =\{j \in \mathcal{P} \quad \mid \quad(i, j, k) \in \mathcal{P} \text { for some } i, k \in \mathcal{P}\}, \\
\mathcal{B} & =\{i \in \mathcal{P} \quad \mid(i, j, k) \in \mathcal{P} \text { for some } j, k \in \mathcal{P}\},
\end{aligned}
$$

so that by our assumption $\mathcal{P}=\mathcal{T} \cup \mathcal{M} \cup \mathcal{B}$. The group $U_{\mathcal{P}}$ consists of matrices of the form

$$
u(A, C, B)=\left(\begin{array}{ccc}
I d_{|\mathcal{B}|} & A & C \\
0 & I d_{|\mathcal{M}|} & B \\
0 & 0 & I d_{|\mathcal{T}|}
\end{array}\right) .
$$

Note that if $\mathcal{B}<\mathcal{M}<\mathcal{T}$ in $\mathcal{P}$, then $U_{\mathcal{P}}$ is the unipotent radical of the parabolic subgroup

$$
P_{|\mathcal{B}|,|\mathcal{M}|,|\mathcal{T}|}=\left\{\left(\begin{array}{ccc}
A & * & * \\
0 & B & * \\
0 & 0 & C
\end{array}\right) \mid A \in \mathrm{GL}_{|\mathcal{B}|}\left(\mathbb{F}_{q}\right), B \in \mathrm{GL}_{|\mathcal{M}|}\left(\mathbb{F}_{q}\right), C \in \mathrm{GL}_{|\mathcal{T}|}\left(\mathbb{F}_{q}\right)\right\}
$$

of $\mathrm{GL}_{|\mathcal{B}|+|\mathcal{M}|+|\mathcal{T}|}\left(\mathbb{F}_{q}\right)$.

If $u(A, C, B) \in U_{\mathcal{P}}$, then the corresponding two-sided orbit is

$$
\begin{aligned}
\left\{\left(\begin{array}{cccc}
I d_{|\mathcal{B}|} & A & C+ & A X+Y B \\
0 & I d_{|\mathcal{M}|} & B & \\
0 & 0 & I d_{|\mathcal{T}|} &
\end{array}\right) \mid\right. & \left.\left(\begin{array}{ccc}
I d_{|\mathcal{B}|} & 0 & 0 \\
0 & I d_{|\mathcal{M}|} & X \\
0 & 0 & I d_{|\mathcal{T}|}
\end{array}\right),\left(\begin{array}{ccc}
I d_{|\mathcal{B}|} & Y & 0 \\
0 & I d_{|\mathcal{M}|} & 0 \\
0 & 0 & I d_{|\mathcal{T}|}
\end{array}\right) \in U_{\mathcal{P}}\right\} .
\end{aligned}
$$


Similarly, if $\lambda(U, W, V) \in \mathfrak{n}_{\mathcal{P}}^{*}$ corresponds to the matrix $u(U, W, V)$, then the coorbit containing $\lambda(U, W, V)$ is

$$
\begin{aligned}
\left\{\lambda\left(U+W\left(X^{\operatorname{Tr}}\right), W, V+\left(Y^{\operatorname{Tr}}\right) W\right) \mid\right. \\
\left.\left(\begin{array}{ccc}
I d_{|\mathcal{B}|} & 0 & 0 \\
0 & I d_{|\mathcal{M}|}-X \\
0 & 0 & I d_{|\mathcal{T}|}
\end{array}\right),\left(\begin{array}{ccc}
I d_{|\mathcal{B}|} & -Y & 0 \\
0 & I d_{|\mathcal{M}|} & 0 \\
0 & 0 & I d_{|\mathcal{T}|}
\end{array}\right) \in U_{\mathcal{P}}\right\} .
\end{aligned}
$$

Since there are no sequences of length four, the matrix $M_{\phi}^{\eta}=0$, so $\phi$ meshes with $\eta$ if and only if $a_{\phi}^{\eta}=b_{\phi}^{\eta}=0$. But,

$$
\left(a_{\phi}^{\eta}\right)_{i j}= \begin{cases}\sum_{(j, k) \in \mathcal{P}} \phi_{j k} \eta_{i k} & i \in \mathcal{B}, j \in \mathcal{M}, \\ 0 & \text { otherwise }\end{cases}
$$

and

$$
\left(b_{\phi}^{\eta}\right)_{j k}= \begin{cases}\sum_{(i, j) \in \mathcal{P}} \phi_{i j} \eta_{i k} & j \in \mathcal{M}, k \in \mathcal{T}, \\ 0 & \text { otherwise }\end{cases}
$$

which translate into the conditions for $u(A, C, B)$ and $\lambda(U, W, V)$,

$$
A_{* j} \cdot W_{* k}=0 \text { if } j<k \text {, and } B_{j *} \cdot W_{i *}=0 \quad \text { if } i<j,
$$

where $M_{* j}$ is the $j$ th column of $M, M_{i *}$ is the $i$ th row of $M$ and · is the usual vector dot product. For $j \in \mathcal{M}$, let

$$
W^{(j)}=\text { columns } W_{* k} \text { of } W \text { such that } j<k \text { in } \mathcal{P} .
$$

The resulting character formula is

$$
\chi^{\lambda(U, W, V)}(u(A, C, B))= \begin{cases}q^{\sum_{j \in \mathcal{M}} \operatorname{rank}\left(W^{(j)}\right)} \prod_{(i, j) \in \mathcal{P}} \theta\left(\lambda_{i j} u_{i j}\right) & \text { if } \lambda \text { and } u \text { satisfy (5.8) } \\ 0 & \text { otherwise. }\end{cases}
$$

Proposition 5.1 below shows that for these examples supercharacters and superclasses are irreducible characters and conjugacy classes so that (5.9) gives a formula for the irreducible characters of $U_{\mathcal{P}}$.

5.2. Supertheory versus usual character theory. For $\eta \in J^{*}$, let

$$
\begin{aligned}
& \operatorname{ann}_{J}^{R}(\eta)=\left\{\rho \in J^{*} \mid X_{\phi} X_{\rho} \in \operatorname{ker}\left(\lambda_{\eta}\right), \text { for all } \phi \in J^{*}\right\}, \\
& \operatorname{ann}_{J}^{L}(\eta)=\left\{\rho \in J^{*} \mid X_{\rho} X_{\phi} \in \operatorname{ker}\left(\lambda_{\eta}\right), \text { for all } \phi \in J^{*}\right\} .
\end{aligned}
$$

Diaconis and Isaacs give the following characterization of which supercharacters are irreducible characters [10].

Theorem 5.2 (Diaconis, Isaacs). Let $J$ be closed, and $\eta \in J^{*}$. Then $\chi^{\eta}$ is irreducible if and only if $\operatorname{ann}_{J}^{R}(\eta)+\operatorname{ann}_{J}^{L}(\eta)=J^{*}$.

This implies the following more combinatorial proposition.

Proposition 5.1. Let $J \subseteq R^{+}$be a closed subset.

(a) Suppose $\phi \in J^{*}$ and there is no 4-chain $(i, j, k, l) \in \mathcal{P}_{J}$ such that $(i, j),(k, l)$ $\in \operatorname{supp}(\phi)$. Then the two-sided orbit of $x_{\phi}$ is a conjugacy class. 
(b) Suppose $\eta \in J^{*}$ and there is no 4-chain $(i, j, k, l) \in \mathcal{P}_{J}$ such that $(i, k),(j, l)$ $\in \operatorname{supp}(\eta)$. Then $\chi^{\eta}$ is an irreducible character.

Proof. (a) Note that if $\phi$ satisfies the hypothesis, then any $\phi^{\prime} \in O_{\phi}$ satisfies the hypothesis. If the hypothesis is satisfied, then for every $(i, j) \in J$, the group element $x_{i j}(t)$ acts trivially either from the right or from the left. Thus, conjugation by $x_{i j}(t)$ is either a right or left action.

(b) Suppose there is no $(i, j, k, l) \in \mathcal{P}$ such that $(i, k),(j, l) \in \operatorname{supp}(\eta)$, and let $\rho \in J^{*}$. By Theorem [5.2, it suffices to find $\rho^{\prime} \in \operatorname{ann}_{J}^{R}(\eta)$ and $\rho^{\prime \prime} \in \operatorname{ann}_{J}^{L}(\eta)$ such that $\rho=\rho^{\prime}+\rho^{\prime \prime}$. Let

$$
\begin{aligned}
& \rho_{j k}^{\prime}= \begin{cases}0 & \text { if there exists }(i, j, k) \in \mathcal{P} \text { with }(i, k) \in \operatorname{supp}(\eta), \\
\rho_{j k} & \text { otherwise }\end{cases} \\
& \rho_{j k}^{\prime \prime}= \begin{cases}0 & \text { if there exists }(j, k, l) \in \mathcal{P} \text { with }(j, l) \in \operatorname{supp}(\eta) \text { OR } \rho_{j k}^{\prime} \neq 0, \\
\rho_{j k} & \text { otherwise }\end{cases}
\end{aligned}
$$

Note that by the assumption, if $\rho_{j k} \neq 0$, then either $\rho_{j k}^{\prime} \neq 0$ or $\rho_{j k}^{\prime \prime} \neq 0$. Thus, $\rho=\rho^{\prime}+\rho^{\prime \prime}$.

For $\phi \in J^{*}$,

$$
X_{\phi} X_{\rho^{\prime}}=-X_{\phi}+X_{\phi} x_{\rho^{\prime}} .
$$

If $X_{\phi} X_{\rho^{\prime}}=X_{\phi^{\prime}}$, then by Theorem 4.1

so

$$
\phi_{i l}^{\prime}=\sum_{(i, j, l) \in \mathcal{P}} \phi_{i j} \rho_{j l}^{\prime}
$$

$$
\lambda^{\eta}\left(X_{\phi} X_{\rho^{\prime}}\right)=\sum_{(i, k)} \eta_{i k} \sum_{(i, j, k)} \phi_{i j} \rho_{j k}^{\prime}=\sum_{(i, j)} \phi_{i j}\left(\sum_{(i, j, k)} \eta_{i k} \rho_{j k}^{\prime}\right)=0 .
$$

Thus, $\rho^{\prime} \in \operatorname{ann}_{J}^{R}(\eta)$. Similarly, $\rho^{\prime \prime} \in \operatorname{ann}_{J}^{L}(\eta)$, so by Theorem 5.2, $\chi^{\eta}$ is an irreducible character.

Corollary 5.1. If $\mathcal{P}_{J}$ has no 4-chains, then all superclasses are conjugacy classes, and all supercharacters are irreducible characters.

The following corollary uses [10] and the fact that we may choose our co-orbit representatives to satisfy (5.7) to obtain a full characterization of when supercharacters are irreducible.

Corollary 5.2. Let $J=R^{+}$so that $U_{J}=U_{n}\left(\mathbb{F}_{q}\right)$. Let $\eta \in J^{*}$ such that $\eta$ satisfies (5.7). Then $\chi^{\eta}$ is an irreducible character if and only if there is no 4$\operatorname{chain}(i, j, k, l) \in \mathcal{P}_{J}$ such that $(i, k),(j, l) \in \operatorname{supp}(\eta)$.

Proof. Consider

$$
\begin{aligned}
\operatorname{ann}_{J}^{R}(\eta) & =\left\{\rho \in J^{*} \mid \sum_{(i, k) \in \operatorname{supp}(\eta)} \eta_{i k}\left(\sum_{(i, j, k)} \phi_{i j} \rho_{j k}\right)=0, \text { for all } \phi \in J^{*}\right\} \\
& =\left\{\rho \in J^{*} \mid \sum_{k \geq j} \eta_{i k} \rho_{j k}=0, \text { for all }(i, j) \in J\right\}, \\
\operatorname{ann}_{J}^{L}(\eta) & =\left\{\rho \in J^{*} \mid \sum_{(i, k) \in \operatorname{supp}(\eta)} \eta_{i k}\left(\sum_{(i, j, k)} \rho_{i j} \phi_{j k}\right)=0, \text { for all } \phi \in J^{*}\right\} \\
& =\left\{\rho \in J^{*} \mid \sum_{i \leq j} \eta_{i k} \rho_{i j}=0, \text { for all }(j, k) \in J\right\} .
\end{aligned}
$$


By assumption, $(i, j),(k, l) \in \operatorname{supp}(\eta)$ implies that $i \neq k$ and $j \neq l$, so

$$
\begin{aligned}
& \operatorname{ann}_{J}^{R}(\eta)=\left\{\rho \in J^{*} \mid \eta_{i k} \rho_{j k}=0, \text { for all }(i, j, k) \in \mathcal{P},(i, k) \in \operatorname{supp}(\eta)\right\}, \\
& \operatorname{ann}_{J}^{L}(\eta)=\left\{\rho \in J^{*} \mid \eta_{i k} \rho_{i j}=0, \text { for all }(i, j, k) \in \mathcal{P},(i, k) \in \operatorname{supp}(\eta)\right\} .
\end{aligned}
$$

Thus,

$$
\operatorname{ann}_{J}^{R}(\eta)+\operatorname{ann}_{J}^{L}(\eta)=J^{*}
$$

if and only if there does not exist $(i, j, k) \in \mathcal{P}$ and $(j, k, l) \in \mathcal{P}$ such that $(i, k),(j, l) \in$ $\operatorname{supp}(\eta)$.

Examples. (1) Corollary 5.1 implies that the supertheory for the Heisenberg group and the pattern groups of Section 5, Example 3, is just the ordinary irreducible character theory.

(2) If $\phi, \eta \in J^{*}$ satisfy $|\operatorname{supp}(\phi)|=|\operatorname{supp}(\eta)|=1$, then $x_{\phi}$ is a conjugacy class and $\chi^{\eta}$ is an irreducible character.

(3) If $U_{\mathcal{P}}=U_{n}\left(\mathbb{F}_{q}\right)$, then the supercharacters $\chi^{\eta}$ with maximal degree are given by $\operatorname{supp}(\eta)=\{(i, n+1-i) \quad \mid 1 \leq i \leq\lfloor n / 2\rfloor\}$, or in terms of the poset, $\operatorname{supp}(\eta)$ is the set of pairs

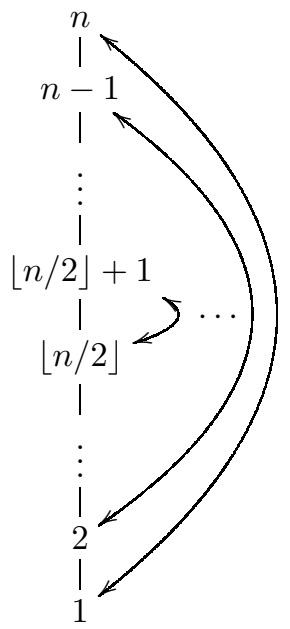

While the poset contains $i<j<k<l$, there is no such sequence such that $(i, k),(j, l) \in \operatorname{supp}(\eta)$. Thus, Proposition 5.1 implies $\chi^{\eta}$ is an irreducible character. Note that since the degrees of the supercharacters are greater than the degrees of the irreducible characters, we have that all the irreducible characters of maximal degree are supercharacters of this form.

These characters were first identified by Lehrer [13]. André [4] and Yan [17. realized that they are in fact supercharacters. The fact that they constitute all of the characters of maximal degree was first proved by Isaacs [12.

Note that if $x_{\phi}$ is such that $\operatorname{supp}(\phi)=\{(i, n+1-i) \mid 1 \leq i \leq\lfloor n / 2\rfloor\}$, then $x_{\phi}$ is also a conjugacy class.

(Counter) Examples. The following examples show that while the conditions in Proposition 5.1 are necessary, they are not sufficient. 
1. One can show that if

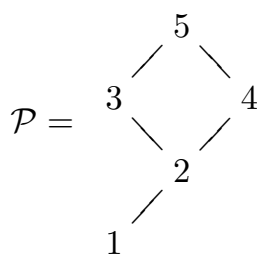

and $\operatorname{supp}(\phi)=\{(1,2),(2,4),(3,5)\}$, then $O_{\phi}$ is a conjugacy class (even though $(1,2,3,5) \in \mathcal{P})$. See [3] for a necessary and sufficient condition in the case when $\mathcal{P}$ is a chain.

2. Suppose

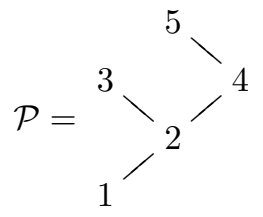

and $\operatorname{supp}(\eta)=\{(1,3),(1,4),(2,5)\}$. Then,

$$
\begin{aligned}
\operatorname{ann}_{J}^{R}(\eta) & =\left\{\rho^{\prime} \in J^{*} \mid \eta_{13} \phi_{12} \rho_{23}^{\prime}+\eta_{14} \phi_{12} \rho_{24}^{\prime}+\eta_{25} \phi_{24} \rho_{45}^{\prime}=0, \text { for all } \phi_{12}, \phi_{24} \in \mathbb{F}_{q}\right\} \\
& =\left\{\rho^{\prime} \in J^{*} \mid \rho_{45}^{\prime}=0, \rho_{23}^{\prime}=-\eta_{13}^{-1} \rho_{24}^{\prime} \eta_{14}\right\}, \\
\operatorname{ann}_{J}^{R}(\eta) & =\left\{\rho^{\prime \prime} \in J^{*} \mid \eta_{13} \rho_{12}^{\prime \prime} \phi_{23}+\eta_{14} \rho_{12}^{\prime \prime} \phi_{24}+\eta_{25} \rho_{24}^{\prime \prime} \phi_{45}=0,\right. \\
& =\left\{\rho^{\prime \prime} \in J^{*} \mid \rho_{12}^{\prime \prime}=\rho_{24}^{\prime \prime}=0\right\} .
\end{aligned}
$$

Given $\rho \in J^{*}$, define $\rho^{\prime} \in \operatorname{ann}_{J}^{R}(\eta)$ and $\rho^{\prime \prime} \in \operatorname{ann}_{J}^{L}(\eta)$ by

$$
\begin{aligned}
& \rho_{i j}^{\prime}= \begin{cases}\rho_{i j} & \text { if }(i, j) \notin\{(2,3),(4,5)\} \\
0 & \text { if }(i, j)=(4,5), \\
-\eta_{13}^{-1} \rho_{24} \eta_{14} & \text { if }(i, j)=(2,3)\end{cases} \\
& \rho_{i j}^{\prime \prime}= \begin{cases}0 & \text { if }(i, j) \notin\{(2,3),(4,5)\} \\
\rho_{45} & \text { if }(i, j)=(4,5), \\
\rho_{23}+\eta_{13}^{-1} \rho_{24} \eta_{14} & \text { if }(i, j)=(2,3) .\end{cases}
\end{aligned}
$$

Since $\rho=\rho^{\prime}+\rho^{\prime \prime}$, Theorem 5.2 implies that $\chi^{\eta}$ is an irreducible character.

3. This example shows that determining whether a supercharacter $\chi^{\eta}$ is irreducible can require more information than just the support of $\eta$. Suppose

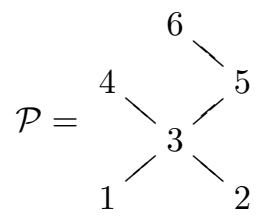


and $\operatorname{supp}(\eta)=\{(1,4),(1,5),(2,4),(2,5),(3,6)\}$. Then,

$$
\begin{aligned}
& \operatorname{ann}_{J}^{R}(\eta)=\left\{\rho \in J^{*} \mid \eta_{14} \phi_{13} \rho_{34}+\eta_{15} \phi_{13} \rho_{35}+\eta_{24} \phi_{23} \rho_{34}+\eta_{25} \phi_{23} \rho_{35}\right. \\
& \left.+\eta_{36} \phi_{35} \rho_{56}=0, \phi \in J^{*}\right\} \\
& =\left\{\rho \in J^{*} \mid \rho_{56}=0, \eta_{14} \rho_{34}=-\eta_{15} \rho_{35}, \eta_{24} \rho_{34}=-\eta_{25} \rho_{35}\right\}, \\
& \operatorname{ann}_{J}^{R}(\eta)=\left\{\rho \in J^{*} \mid \eta_{14} \rho_{13} \phi_{34}+\eta_{15} \rho_{13} \phi_{35}+\eta_{24} \rho_{23} \phi_{34}+\eta_{25} \rho_{23} \phi_{34}\right. \\
& \left.+\eta_{36} \rho_{35} \phi_{56}=0, \phi \in J^{*}\right\} \\
& =\left\{\rho \in J^{*} \mid \rho_{35}=0, \eta_{14} \rho_{13}=-\eta_{24} \rho_{23}, \eta_{15} \rho_{13}=-\eta_{25} \rho_{23}\right\} .
\end{aligned}
$$

Let $\rho \in J^{*}$. We want $\rho=\rho^{\prime}+\rho^{\prime \prime}$ for $\rho^{\prime} \in \operatorname{ann}_{J}^{R}(\eta)$ and $\rho^{\prime \prime} \in \operatorname{ann}_{J}^{L}(\eta)$. Since elements of $\operatorname{ann}_{J}^{R}(\eta)$ can have arbitrary values at $J \backslash\{(5,6),(3,4),(3,5)\}$, and elements of $\operatorname{ann}_{J}^{L}(\eta)$ can have arbitrary values at $(5,6)$ and $(3,4)$, we can find $\rho^{\prime}$ and $\rho^{\prime \prime}$ such that $\rho_{i j}=\rho_{i j}^{\prime}+\rho_{i j}^{\prime \prime}$ for $(i, j) \neq(3,5)$. However, $\rho_{35}^{\prime \prime}=0$, so

$$
\rho_{35}=\rho_{35}^{\prime}=-\eta_{15}^{-1} \eta_{14} \rho_{34}^{\prime}=-\eta_{25}^{-1} \eta_{24} \rho_{34}^{\prime},
$$

which can happen if and only if $\eta_{25} \eta_{14}-\eta_{15} \eta_{24}=0$. Thus, $\chi^{\eta}$ is an irreducible character if and only if $\eta_{25} \eta_{14}-\eta_{15} \eta_{24}=0$.

\section{An Algebra group supercharacter Formula}

Let $H \subseteq U_{n}\left(\mathbb{F}_{q}\right)$ be an algebra group with corresponding vector space $V_{H}$ (as in Proposition 2.2), and let $\mathfrak{n}_{H}=H-1$. This section gives a formula for a general supercharacter on a general superclass. The main result is followed by a corollary regarding supercharacter values and by several examples.

Fix a basis $\left\{v_{1}, v_{2}, \ldots, v_{d}\right\}$ for $V_{H}$ and let $\left\{\lambda_{1}, \lambda_{2}, \ldots, \lambda_{d}\right\}$ be a dual basis for the dual space $V_{H}^{*}$. For $\phi=\left(\phi_{1}, \phi_{2}, \ldots, \phi_{d}\right) \in \mathbb{F}_{q}^{d}$, let $x_{\phi} \in H$ be the element that corresponds to the vector

$$
\phi_{1} v_{1}+\phi_{2} v_{2}+\cdots+\phi_{d} v_{d} \in V_{H} .
$$

Similarly, for $\eta=\left(\eta_{1}, \eta_{2}, \ldots, \eta_{d}\right) \in \mathbb{F}_{q}^{d}$, let $\lambda_{\eta} \in \mathfrak{n}_{H}^{*}$ be the functional corresponding to

$$
\eta_{1} \lambda_{1}+\eta_{2} \lambda_{2}+\cdots+\eta_{d} \lambda_{d} \in V_{H}^{*}
$$

Let $c_{i j}^{k} \in \mathbb{F}_{q}$ be defined by

$$
X_{v_{i}} X_{v_{j}}=\sum_{k=1}^{d} c_{i j}^{k} X_{v_{k}},
$$

where $1+X_{v_{i}} \in H$ is the group element corresponding to the basis vector $v_{i} \in V_{H}$. Let $C_{i}$ and $C^{j}$ denote the $d \times d$ matrices given by

$$
\left(C_{i}\right)_{j k}=c_{i j}^{k} \quad \text { and } \quad\left(C^{j}\right)_{i k}=c_{i j}^{k} .
$$

For $\phi, \eta \in \mathbb{F}_{q}^{d}$, let $M_{\phi}^{\eta}$ be the $d \times d$ matrix given by

$$
\left(M_{\phi}^{\eta}\right)_{i j}=\phi C_{i} C^{j} \eta
$$

Define $a_{\phi}^{\eta}, b_{\phi}^{\eta} \in \mathbb{F}_{q}^{d}$ by

$$
\begin{aligned}
& \left(a_{\phi}^{\eta}\right)_{i}=\phi C_{i} \eta \\
& \left(b_{\phi}^{\eta}\right)_{j}=\phi C^{j} \eta
\end{aligned}
$$


We say that $\phi$ meshes with $\eta$ if

(1) there exists $b \in \mathbb{F}_{q}^{d}$ such that $M_{\phi}^{\eta} b=-a_{\phi}^{\eta}$,

(2) $b_{\phi}^{\eta}$ is perpendicular to $\operatorname{Null}\left(M_{\phi}^{\eta}\right)$.

Theorem 6.1. Let $H$ be an algebra group with $\operatorname{dim}\left(V_{H}\right)=d$. Let $\phi, \eta \in \mathbb{F}_{q}^{d}$ so that $x_{\phi} \in H$ and $\lambda_{\eta} \in \mathfrak{n}_{H}^{*}$ are defined according to the bases of $V_{H}$ and $V_{H}^{*}$, respectively. Let $M_{\phi}^{\eta}, a_{\phi}^{\eta}$, and $b_{\phi}^{\eta}$ be as in (6.1), (6.2), and (6.3), respectively. Then

$$
\overline{\chi^{\eta}\left(x_{\phi}\right)}= \begin{cases}q^{\operatorname{corank}(\eta)-\operatorname{rank}\left(M_{\phi}^{\eta}\right)} \theta\left(b_{0} \cdot b_{\phi}^{\eta}\right) \prod_{i=1}^{d} \theta\left(\phi_{i} \eta_{i}\right) & \text { if } \phi \text { meshes with } \eta, \\ 0 & \text { otherwise, }\end{cases}
$$

where $b_{0} \in \mathbb{F}_{q}^{d}$ satisfies $M_{\phi}^{\eta} b_{0}=-a_{\phi}^{\eta}$, and $q^{\operatorname{corank}(\eta)}$ is the size of the right $H$-orbit of $\lambda_{\eta}$.

Proof. From the definitions in Section 2.3,

$$
\overline{\chi^{\eta}\left(x_{\phi}\right)}=\frac{q^{\operatorname{corank}(\eta)}}{\left|O_{\phi}\right|} \sum_{\rho \in \mathbb{F}_{q}^{d}} \theta \circ \lambda_{\eta}\left(X_{\rho}\right)=\frac{q^{\operatorname{corank}(\eta)}}{q^{2 d}} \sum_{a, b \in \mathbb{F}_{q}^{d}} \theta \circ \lambda_{\eta}\left(x_{a} X_{\phi} x_{b}\right) .
$$

Note that for $a=\left(a_{1}, a_{2}, \ldots, a_{d}\right), b=\left(b_{1}, b_{2}, \ldots, b_{d}\right) \in \mathbb{F}_{q}^{d}$,

$$
x_{a} X_{\phi} x_{b}=\sum_{i=1}^{d} \phi_{i} X_{v_{i}}+\sum_{i, j, k}\left(a_{i} \phi_{j}+\phi_{i} b_{j}\right) c_{i j}^{k} X_{v_{k}}+\sum_{i, j, k, l, m} a_{i} \phi_{j} b_{l} c_{i j}^{k} c_{k l}^{m} X_{v_{m}} .
$$

Thus,

$$
\begin{aligned}
\overline{\chi^{\eta}\left(x_{\phi}\right)}=\frac{q^{\operatorname{corank}(\eta)}}{q^{2 d}} \sum_{a, b \in \mathbb{F}_{q}^{d}} \theta\left(\sum_{m=1}^{d} \eta_{m}(\right. & \phi_{m}+\sum_{i, j}\left(a_{i} \phi_{j}+\phi_{i} b_{j}\right) c_{i j}^{m} \\
& \left.\left.+\sum_{i, j, k, l} a_{i} \phi_{j} b_{l} c_{i j}^{k} c_{k l}^{m}\right)\right) \\
= & \frac{q^{\operatorname{corank}(\eta)} \theta_{\phi \eta}}{q^{2 d}} \sum_{a, b \in \mathbb{F}_{q}^{d}} \theta\left(\sum_{m=1}^{d} \eta_{m}\left(\sum_{i, j}\left(a_{i} \phi_{j}+\phi_{i} b_{j}\right) c_{i j}^{m}+\sum_{i, j, k, l} a_{i} \phi_{j} b_{l} c_{i j}^{k} c_{k l}^{m}\right)\right),
\end{aligned}
$$

where

$$
\theta_{\phi \eta}=\theta\left(\phi_{1} \eta_{1}+\phi_{2} \eta_{2}+\cdots+\phi_{d} \eta_{d}\right) .
$$

Collect all terms involving $a_{i}$ to obtain

$$
\begin{aligned}
\overline{\chi^{\eta}\left(x_{\phi}\right)}= & \frac{q^{\operatorname{corank}(\eta)} \theta_{\phi \eta}}{q^{2 d}} \sum_{a, b \in \mathbb{F}_{q}^{d}} \theta\left(\sum_{i=1}^{d} a_{i}\left(\sum_{j, m} \phi_{j} c_{i j}^{m} \eta_{m}+\sum_{j, k, l, m} \phi_{j} c_{i j}^{k} c_{k l}^{m} \eta_{m} b_{l}\right)\right. \\
= & \left.+\sum_{i, j, m} \phi_{i} c_{i j}^{m} \eta_{m} b_{j}\right) \\
q^{2 d} & \sum_{a, b \in \mathbb{F}_{q}^{d}} \theta\left(a \cdot\left(a_{\phi}^{\eta}+M_{\phi}^{\eta} b\right)+b_{\phi}^{\eta} \cdot b\right) .
\end{aligned}
$$


Note that

$$
\sum_{a \in \mathbb{F}_{q}^{d}} \theta\left(a \cdot\left(a_{\phi}^{\eta}+M_{\phi}^{\eta} b\right)\right)=0
$$

unless $M_{\phi}^{\eta} b=-a_{\phi}^{\eta}$. Thus, if $\mathcal{S}=\left\{b \in \mathbb{F}_{q}^{d} \mid M_{\phi}^{\eta} b=-a_{\phi}^{\eta}\right\}$, then

$$
\overline{\chi^{\eta}\left(x_{\phi}\right)}=\frac{q^{\operatorname{corank}(\eta)} \theta_{\phi \eta}}{q^{d}} \sum_{b \in \mathcal{S}} \theta\left(b_{\phi}^{\eta} \cdot b\right) .
$$

Fix an element $b_{0} \in \mathcal{S}$. Since every other vector in $\mathcal{S}$ is of the form $b_{0}+b^{\prime}$, where $b^{\prime}$ is in the nullspace of $M_{\phi}^{\eta}$, we have

$$
\overline{\chi^{\eta}\left(x_{\phi}\right)}=\frac{q^{\operatorname{corank}(\eta)} \theta_{\phi \eta} \theta\left(b_{0} \cdot b_{\phi}^{\eta}\right)}{q^{d}} \sum_{b^{\prime} \in \operatorname{Null}\left(M_{\phi}^{\eta}\right)} \theta\left(b^{\prime} \cdot b_{\phi}^{\eta}\right) .
$$

Let $\left\{b_{1}^{\prime}, b_{2}^{\prime}, \ldots, b_{r}^{\prime}\right\}$ be a basis for $\operatorname{Null}\left(M_{\phi}^{\eta}\right)$. Then

$$
\sum_{b^{\prime} \in \operatorname{Null}\left(M_{\phi}^{\eta}\right)} \theta\left(b^{\prime} \cdot b_{\phi}^{\eta}\right)=\sum_{t \in \mathbb{F}_{q}^{r}} \prod_{i=1}^{r} \theta\left(t_{i} b_{i}^{\prime} \cdot b_{\phi}^{\eta}\right)=\prod_{i=1}^{r} \sum_{t_{i} \in \mathbb{F}_{q}} \theta\left(t_{i} b_{i}^{\prime} \cdot b_{\phi}^{\eta}\right) .
$$

Thus, if any $b_{i}^{\prime}$ is not orthogonal to $b_{\phi}^{\eta}$, then the product is zero. If $\phi$ meshes with $\eta$, then

$$
\overline{\chi^{\eta}\left(x_{\phi}\right)}=\frac{q^{\operatorname{corank}(\eta)} \theta_{\phi \eta} \theta\left(b_{0} \cdot b_{\phi}^{\eta}\right)}{q^{d}} q^{d-\operatorname{rank}\left(M_{\phi}^{\eta}\right)}=q^{\operatorname{corank}(\eta)-\operatorname{rank}\left(M_{\phi}^{\eta}\right)} \theta_{\phi \eta} \theta\left(b_{0} \cdot b_{\phi}^{\eta}\right),
$$

as desired.

Corollary 6.1. Let $H \subseteq U_{n}\left(\mathbb{F}_{q}\right)$ be an algebra group and suppose $\operatorname{char}\left(\mathbb{F}_{q}\right)=p$. Then

(a) The nonzero supercharacter values are integer multiples of pth roots of unity.

(b) If $p=2$, then all supercharacters are real-valued.

Remarks. (1) Note that if $H=U_{J}$ is a pattern group, then a natural basis of $V_{H}$ is $\left\{v^{(i j)} \mid(i, j) \in J\right\}$, where $v^{(i j)} \in \mathbb{F}_{q}^{|J|}$ is given by $v_{r s}^{(i j)}=\delta_{i j, r s}$. With this basis, Theorem 6.1 reduces to Theorem 5.1 .

(2) Corollary 6.1 (a) is the supercharacter version of a conjecture by Isaacs [12, Conjecture 10.1] concerning irreducible characters.

\subsection{Examples.}

1. A supercharacter formula. Let $H_{n-1}=1+X \mathbb{F}_{q}[X] /\left(X^{n-1}\right)$ be the abelian group of polynomials that begin with 1 under polynomial multiplication $\bmod X^{n-1}$. The supercharacters for this algebra group were computed as an example in [10, Appendix C]. We extend these calculations by considering the semi-direct product of $H_{n-1}$ with $\mathbb{F}_{q}^{n-1}$, the abelian group under vector addition.

For $f=1+\sum_{i=2}^{n-1} a_{i} X^{i-1} \in H_{n-1}, t=\left(t_{1}, t_{2}, \ldots, t_{n-1}\right) \in \mathbb{F}_{q}^{n-1}$, define $f t f^{-1}=$ $\left(t_{1}^{\prime}, t_{2}^{\prime}, \ldots, t_{n-2}^{\prime}\right) \in \mathbb{F}_{q}^{n-1}$ by

$$
t_{j}^{\prime}=t_{j}+\sum_{k=j+1}^{n-1} a_{k} t_{k}
$$


This action makes $H=H_{n-1} \ltimes \mathbb{F}_{q}^{n-1}$ an algebra group. In fact, as a subgroup of $U_{n}$,

$$
H=\left\{\left(\begin{array}{ccccc|c}
1 & a_{2} & a_{3} & \cdots & a_{n-1} & t_{1} \\
0 & \ddots & \ddots & \ddots & \vdots & \vdots \\
& & 1 & a_{2} & a_{3} & t_{n-3} \\
\vdots & & \ddots & 1 & a_{2} & t_{n-2} \\
0 & 0 & \cdots & 0 & 1 & t_{n-1} \\
\hline 0 & 0 & \cdots & 0 & 0 & 1
\end{array}\right) \mid a_{2}, \ldots, a_{n-1}, t_{1}, \ldots, t_{n-1} \in \mathbb{F}_{q}\right\}
$$

Let $\left\{v_{2}, v_{3}, \ldots, v_{n-1}, v_{\overline{1}}, v_{\overline{2}}, \ldots, v_{n-1}\right\}$ be the basis of $V_{H}$ given by

$X_{v_{i}}=X \in \mathfrak{n}$, where $X_{1 i}=X_{2, i+1}=\cdots=X_{n-i, n-1}=1$ with zeroes elsewhere, $X_{v_{\bar{j}}}=X \in \mathfrak{n}$, where $X_{j n}=1$ with zeroes elsewhere.

Let $\left\{\lambda_{2}, \ldots, \lambda_{n-1}, \lambda_{\overline{1}}, \ldots, \lambda_{\overline{n-1}}\right\}$ be the corresponding dual basis of $V_{H}^{*}$.

A small computation shows that the superclasses are indexed by

$\left\{(i, \bar{j}, a, t),(i, \cdot, a, 0),(\cdot, \bar{j}, 0, t),(\cdot, \cdot, 0,0) \mid 2 \leq i \leq n-1, n-i<j \leq n-1, a, t \in \mathbb{F}_{q}^{\times}\right\}$,

such that natural representatives for the orbits $O_{(i, \bar{j}, a, t)}, O_{(i, \cdot, a, 0)}, O_{(\cdot, \bar{j}, 0, t)}$, and $O_{(\cdot, \cdot, 0,0)}$ correspond to the vectors

$$
a v_{i}+b v_{\bar{j}}, \quad a v_{i}, \quad b v_{\bar{j}}, \quad 0, \quad \text { respectively. }
$$

Similarly, the co-orbits are indexed by the same quadruplets such that natural representatives for the orbits $O^{(i, \bar{j}, a, t)}, O^{(i, \cdot, a, 0)}, O^{(\cdot, \bar{j}, 0, t)}$, and $O^{(\cdot, \cdot, 0,0)}$ are the functionals corresponding to the vectors

$$
a \lambda_{i}+b \lambda_{\bar{j}}, \quad a \lambda_{i}, \quad b \lambda_{\bar{j}}, \quad 0, \quad \text { respectively }
$$

We first calculate the basic ingredients $M_{\phi}^{\eta}, a_{\phi}^{\eta}$, and $b_{\phi}^{\eta}$ of (6.1), (6.2), and (6.3). Note that

$$
\begin{array}{ll}
c_{i j}^{k}= \begin{cases}1 & \text { if } i+j \leq k, k \in\{2, \ldots, n-1\}, \\
0 & \text { otherwise }\end{cases} & c_{\overline{i j}}^{k}=0, \\
c_{i \bar{j}}^{k}= \begin{cases}1 & \text { if } k=j-i+1, k \in\{\overline{1}, \ldots, \overline{n-1}\}, \\
0 & \text { otherwise }\end{cases} & c_{\overline{i j}}^{k}=0
\end{array}
$$

so that by the definition (6.1), for $a, b, s, t \in \mathbb{F}_{q}$, the $(f, g)$ entry of $M_{(i, \bar{j}, a, s)}^{(k, \bar{l}, b, t)}$ is

$$
\left(a c_{f i}^{2}+s c_{f \bar{j}}^{2}, \ldots, a c_{f i}^{n-1}+s c_{f \bar{j}}^{n-1}, a c_{f i}^{\overline{1}}+s c_{f \bar{j}}^{\overline{1}}, \ldots, a c_{f i}^{\overline{n-1}}+s c_{f \bar{j}}^{\overline{n-1}}\right)\left(\begin{array}{c}
b c_{2 g}^{k}+t c_{2 g}^{\bar{l}} \\
\vdots \\
b c_{n-1, g}^{k}+t c_{n-1, g}^{\bar{l}} \\
0 \\
\vdots \\
0
\end{array}\right)
$$

$=0, \quad$ unless $f \in\{2, \ldots, n-1\}$. 
If $f, g \in\{2, \ldots, n-1\}$, then

$$
\begin{aligned}
\left(M_{(i, \bar{j}, a, s)}^{(k, \bar{l}, b, t)}\right)_{f, g} & =\left(0, \ldots, 0, a c_{f i}^{f+i}, 0, \ldots, 0, s c_{f \bar{j}}^{\overline{j-f+1}}, 0, \ldots, 0\right)\left(\begin{array}{c}
0 \\
\vdots \\
0 \\
b c_{k-g, g}^{k} \\
0 \\
\vdots \\
0
\end{array}\right) \\
& = \begin{cases}a b & \text { if } k-g=f+i, \\
0 & \text { otherwise. }\end{cases}
\end{aligned}
$$

If $f \in\{2, \ldots, n-1\}$ and $g \in\{\overline{1}, \ldots, \overline{n-1}\}$, then

$$
\begin{aligned}
\left(M_{(i, \bar{j}, a, s)}^{(k, \bar{l}, b, t)}\right)_{f, g} & =\left(0, \ldots, 0, a c_{f i}^{f+i}, 0, \ldots, 0, s c_{f \bar{j}}^{\overline{j-f+1}}, 0, \ldots, 0\right)\left(\begin{array}{c}
0 \\
\vdots \\
0 \\
t c_{g-l+1, g}^{\bar{l}} \\
0 \\
\vdots \\
0
\end{array}\right) \\
& = \begin{cases}a t & \text { if } g-l+1=f+i, \\
0 & \text { otherwise. }\end{cases}
\end{aligned}
$$

Similarly,

$$
\begin{aligned}
& \left(a_{(i, \bar{j}, a, s)}^{(k, \bar{l}, b, t)}\right)_{f}= \begin{cases}\delta_{i+f, k} a b+\delta_{j-f-1, l} s t & \text { if } f \in\{2, \ldots, n-1\}, \\
0 & \text { if } f \in\{\overline{1}, \ldots, \overline{n-1}\},\end{cases} \\
& \left(b_{(i, \bar{j}, a, s)}^{(k, \bar{l}, b, t)}\right)_{g}= \begin{cases}\delta_{k-g, i} a b & \text { if } g \in\{2, \ldots, n-1\} \\
\delta_{g-l+1, i} a t & \text { if } g \in\{\overline{1}, \ldots, \overline{n-1}\} .\end{cases}
\end{aligned}
$$

Note that if $b_{(i, \bar{j}, a, s)}^{(k, \bar{l}, b, t)}$ has a nonzero entry in row $g$, then $M_{(i, \bar{j}, a, s)}^{(k, \bar{l}, b, t)}$ has no nonzero entry in column $g$, so in order for $b_{(i, \bar{j}, a, s)}^{(k, \bar{l}, b, t)}$ to be perpendicular to $\operatorname{Null}\left(M_{(i, \bar{j}, a, s)}^{(k, \bar{l}, b, t)}\right)$, we have $b_{(i, \bar{j}, a, s)}^{(k, \bar{l}, b, t)}=0$.

Thus, $(i, \bar{j}, a, s)$ meshes with $(k, \bar{l}, b, t)$ if and only if

(1) $k-i \geq 2$ implies $a=0$ or $b=0$,

(2) $n-1 \geq i+l-1$ implies $a=0$ or $t=0$,

(3) $j-l-1 \geq 2$ implies $s=0$ or $t=0$.

In other words, $(i, \bar{j}, a, s)$ meshes with $(k, \bar{l}, b, t)$ if $a_{(i, \bar{j}, a, s)}^{(k, \bar{l}, b, t)}=b_{(i, \bar{j}, a, s)}^{(k, \bar{l}, b, t)}=0$ (and in this case $\left.M_{(i, \bar{j}, a, s)}^{(k, \bar{l}, b, t)}=0\right)$. Therefore, our supercharacter formula is

$$
\overline{\chi^{(k, \bar{l}, b, t)}\left(x_{(i, \bar{j}, a, s)}\right)}= \begin{cases}q^{\max \{k-2, n-1-l\}} \theta\left(a b \delta_{i k}+s t \delta_{j l}\right) & \text { if } a_{(i, \bar{j}, a, s)}^{(k, \bar{l}, b)}=b_{(i, \bar{j}, a, s)}^{(k, \bar{l}, b, t)}=0, \\ 0 & \text { otherwise, }\end{cases}
$$

where if $i=\cdot, j=\cdot, \bar{k}=\cdot$, or $\bar{l}=\cdot$, then substitute $i=2, j=2, k=n-1$, or $l=n-1$, respectively, in the formula. Note that if we restrict this formula to the subgroup $H_{n-1}$ we obtain the supercharacter formula for $H_{n-1}$ computed in [10]. 
2. A supercharacter table. The following example was used for several examples (and counterexamples) in [10]. Let

$$
H=\left\{\left(\begin{array}{cccc}
1 & a & b & d \\
0 & 1 & a & c \\
0 & 0 & 1 & a \\
0 & 0 & 0 & 1
\end{array}\right) \mid a, b, c, d \in \mathbb{F}_{2}\right\}
$$

which is a group of order 16 with a presentation given by

$$
\left\langle x, r, z \mid x^{4}=r^{2}=z^{2}=1,[x, r]=z,[x, z]=1,[r, z]=1\right\rangle,
$$

where

$$
x=\left(\begin{array}{llll}
1 & 1 & 0 & 0 \\
0 & 1 & 1 & 0 \\
0 & 0 & 1 & 1 \\
0 & 0 & 0 & 1
\end{array}\right), \quad r=\left(\begin{array}{llll}
1 & 0 & 0 & 0 \\
0 & 1 & 0 & 1 \\
0 & 0 & 1 & 0 \\
0 & 0 & 0 & 1
\end{array}\right), \quad z=\left(\begin{array}{llll}
1 & 0 & 0 & 1 \\
0 & 1 & 0 & 0 \\
0 & 0 & 1 & 0 \\
0 & 0 & 0 & 1
\end{array}\right) .
$$

Note that

$$
l=\left(\begin{array}{llll}
1 & 0 & 1 & 0 \\
0 & 1 & 0 & 0 \\
0 & 0 & 1 & 0 \\
0 & 0 & 0 & 1
\end{array}\right)=x^{2} r
$$

(We use a slightly different set of generators than [10] for $H$ ).

The character table of $H$ is

\begin{tabular}{|c|ccccccccccc|}
\hline & $\{1\}$ & $\{z\}$ & $\{l r\}$ & $\{l r z\}$ & $\{x, x z\}$ & $\{x l r, x l r z\}$ & $\{r, r z\}$ & $\{l, l z\}$ & $\{x r, x r z\}$ & $\{x l, x l z\}$ \\
\hline$\chi_{1}$ & 1 & 1 & 1 & 1 & 1 & 1 & 1 & 1 & 1 & 1 \\
$\chi_{2}$ & 1 & 1 & -1 & -1 & $i$ & $-i$ & 1 & -1 & $i$ & $-i$ \\
$\chi_{3}$ & 1 & 1 & 1 & 1 & -1 & -1 & 1 & 1 & -1 & -1 \\
$\chi_{4}$ & 1 & 1 & -1 & -1 & $-i$ & $i$ & 1 & -1 & $-i$ & $i$ \\
$\chi_{5}$ & 1 & 1 & 1 & 1 & 1 & 1 & -1 & -1 & -1 & -1 \\
$\chi_{6}$ & 1 & 1 & -1 & -1 & $i$ & $-i$ & -1 & 1 & $-i$ & $i$ \\
$\chi_{7}$ & 1 & 1 & 1 & 1 & -1 & -1 & -1 & -1 & 1 & 1 \\
$\chi_{8}$ & 1 & 1 & -1 & -1 & $-i$ & $i$ & -1 & 1 & $i$ & $-i$ \\
$\chi_{9}$ & 2 & -2 & 2 & -2 & 0 & 0 & 0 & 0 & 0 & 0 \\
$\chi_{10}$ & 2 & -2 & -2 & 2 & 0 & 0 & 0 & 0 & 0 & 0 \\
\hline
\end{tabular}

The supercharacter table of $H$ is

\begin{tabular}{|c|ccccccc|}
\hline & $\{1\}$ & $\{z\}$ & $\{l r, l r z\}$ & $\{x, x z, x l r, x l r z\}$ & $\{r, r z\}$ & $\{l, l z\}$ & $\{x r, x r z, x l, x l z\}$ \\
\hline$\chi_{1}$ & 1 & 1 & 1 & 1 & 1 & 1 & 1 \\
$\chi_{x}$ & 1 & 1 & 1 & -1 & 1 & 1 & -1 \\
$\chi_{l r}$ & 1 & 1 & 1 & 1 & -1 & -1 & -1 \\
$\chi_{r x l}$ & 1 & 1 & 1 & -1 & -1 & -1 & 1 \\
$\chi_{r}$ & 2 & 2 & -2 & 0 & -2 & 2 & 0 \\
$\chi_{l}$ & 2 & 2 & -2 & 0 & 2 & -2 & 0 \\
$\chi_{z}$ & 4 & -4 & 0 & 0 & 0 & 0 & 0 \\
\hline
\end{tabular}

SO

$$
\begin{gathered}
\chi_{1}=\chi_{1}, \quad \chi_{x}=\chi_{3}, \quad \chi_{l r}=\chi_{5}, \quad \chi_{r x l}=\chi_{7}, \\
\chi_{r}=\chi_{6}+\chi_{8}, \quad \chi_{l}=\chi_{2}+\chi_{4}, \quad \chi_{z}=\chi_{9}+\chi_{10} .
\end{gathered}
$$


There are many more supercharacter theories (in the sense of [10]), and we can find many by inspection, such as

\begin{tabular}{|c|cccccc|}
\hline & $\{1\}$ & $\{z\}$ & $\{l r, l r z\}$ & $\{x, x z, x l r, x l r z, x r, x r z, x l, x l z\}$ & $\{r, r z\}$ & $\{l, l z\}$ \\
\hline$\chi_{1}$ & 1 & 1 & 1 & 1 & 1 & 1 \\
$\chi_{x}$ & 1 & 1 & 1 & -1 & 1 & 1 \\
$\tilde{\chi}_{l r}$ & 2 & 2 & 2 & 0 & -2 & -2 \\
$\chi_{r}$ & 2 & 2 & -2 & 0 & -2 & 2 \\
$\chi_{l}$ & 2 & 2 & -2 & 0 & 2 & -2 \\
$\chi_{z}$ & 4 & -4 & 0 & 0 & 0 & 0 \\
\hline
\end{tabular}

with $\tilde{\chi}_{l r}=\chi_{l r}+\chi_{r x l}$;

\begin{tabular}{|c|cccc|}
\hline & $\{1\}$ & $\{z\}$ & $\{l, r, l r, l z, r z, l r z\}$ & $\{x, x z, x l r, x l r z, x r, x r z, x l, x l z\}$ \\
\hline$\chi_{1}$ & 1 & 1 & 1 & 1 \\
$\chi_{x}$ & 1 & 1 & 1 & -1 \\
$\tilde{\chi}_{l}$ & 6 & 6 & -2 & 0 \\
$\chi_{z}$ & 4 & -4 & 0 & 0 \\
\hline
\end{tabular}

with $\tilde{\chi}_{l}=\chi_{l}+\chi_{r}+\tilde{\chi}_{l r} ;$ or

\begin{tabular}{|c|ccc|}
\hline & $\{1\}$ & $\{z\}$ & $\{l, r, l r, l z, r z, l r z, x, x z, x l r, x l r z, x r, x r z, x l, x l z\}$ \\
\hline$\tilde{\chi}_{1}$ & 1 & 1 & 1 \\
$\tilde{\chi}_{x}$ & 7 & 7 & -1 \\
$\tilde{\chi}_{z}$ & 4 & -4 & 0 \\
\hline
\end{tabular}

with $\tilde{\chi}_{x}=\tilde{\chi}_{l}+\chi_{x}$.

\section{ACKNOWLEDGEMENTS}

We thank Bob Guralnick, Marty Isaacs, and Ning Yan for their help, as well as Eric Marberg and Vidya Venkateswaran for their contributions to examples. We would also like to thank an anonymous referee for his/her comments.

\section{REFERENCES}

[1] André, C. "Basic characters of the unitriangular group," Journal of algebra 175 (1995), 287-319. MR1338979 (96h:20081a)

[2] André, C. "Irreducible characters of finite algebra groups," Matrices and group representations Coimbra, 1998, Textos Mat. Sér B 19 (1999), 65-80. MR1773571 (2001g:20009)

[3] André, C. "The basic character table of the unitriangular group," Journal of Algebra 241 (2001), 437-471. MR.1839342 (2002e:20082)

[4] André, C. "Basic characters of the unitriangular group (for arbitrary primes)," Proceedings of the American Mathematical Society 130 (2002), 1934-1954. MR.1896026 (2003g:20075)

[5] André, C; Neto, A. "Super-characters of finite unipotent groups of types $B_{n}, C_{n}$ and $D_{n}$," J. Algebra 305 (2006), 394-429. MR2264135 (2007j:20013)

[6] André, C; Nicolás, A. "Supercharacters of the adjoint group of a finite radical ring," August 2006 preprint.

[7] Arregi, J; Vera-Lopez, A. "Computing in unitriangular matrices over finite fields." Linear algebra applications 387 (2004), 193-219. MR2069276 (2005c:20082)

[8] Arias-Castro, E; Diaconis, P; Stanley, R. "A super-class walk on upper-triangular matrices," Journal of Algebra 278 (2004), 739-765. MR2071663 (2005f:60101)

[9] Carter, R. Finite groups of Lie type: Conjugacy classes and complex characters. John Wiley and Sons, 1985. MR794307 (87d:20060)

[10] Diaconis, P; Isaacs, M. "Supercharacters and superclasses for algebra groups," Transactions of the American Mathematical Society, 360 (2008), 2359-2392. MR2373317 
[11] Diaconis, P; Saloff-Coste, L. "Comparison techniques for random walk on finite groups," Annals of Probability 21 (1993), 2131-2156. MR1245303 (95a:60009)

[12] Isaacs, M. "Counting characters of upper triangular groups," September 2006 preprint.

[13] Lehrer, G. "Discrete series and the unipotent subgroup," Compositio Mathematica 28 (1974), 9-19. MR0340438(49:5193)

[14] Pierce, R. Associative Algebras, Graduate Texts in Mathematics 88. Studies in the History of Modern Science 9. Springer-Verlag, New York-Berlin, 1982. MR674652 (84c:16001)

[15] Robinson, G. "Counting conjugacy classes of unitriangular groups associated to finitedimensional algebras," Journal of Group Theory 1 (1998), 271-274. MR1633196|(99h:14025)

[16] Spiegel, E; O'Donnell, C. Incidence algebras, Monographs and textbooks in pure mathematics 206, Marcel Dekker, Inc., New York: 1997. MR1445562 (98g:06001)

[17] Yan, N. Representation theory of the finite unipotent linear groups, Unpublished Ph.D. Thesis, Department of Mathematics, Pennsylvania State Unversity, 2001.

[18] Yan, N. "Representations of finite unipotent linear groups by the method of clusters," 2006 preprint.

Department of Mathematics, Stanford University, Stanford, California 94305-4065

Department of Mathematics, Stanford University, 450 Serra Mall, Building 380 , Stanford, California 94305-2125

Current address: Department of Mathematics, University of Colorado, Campus Box 395, Boulder, Colorado 80309-0395 